PHYSICAL REVIEW D 95, 123006 (2017)

\title{
Astrophysical interpretation of the anisotropies in the unresolved gamma-ray background
}

\author{
Shin'ichiro Ando, ${ }^{1, *}$ Mattia Fornasa, ${ }^{1, \dagger}$ Nicolao Fornengo, ${ }^{2,3}$ Marco Regis, ${ }^{2,3}$ and Hannes-S. Zechlin ${ }^{2,3}$ \\ ${ }^{1}$ GRAPPA, University of Amsterdam, Science Park, 1098 XH Amsterdam, Netherlands \\ ${ }^{2}$ Dipartimento di Fisica, Universitá di Torino, via P. Giuria 1, I-10125 Torino, Italy \\ ${ }^{3}$ Istituto Nazionale di Fisica Nucleare, Sezione di Torino, via P. Giuria 1, I-10125 Torino, Italy
}

(Received 27 January 2017; published 13 June 2017)

\begin{abstract}
Recently, a new measurement of the auto- and cross-correlation angular power spectrum (APS) of the isotropic gamma-ray background was performed, based on 81 months of data of the Fermi Large-Area Telescope (LAT). Here, we fit, for the first time, the new APS data with a model describing the emission of unresolved blazars. These sources are expected to dominate the anisotropy signal. The model we employ in our analysis reproduces well the blazars resolved by Fermi LAT. When considering the APS obtained by masking the sources listed in the 3FGL catalog, we find that unresolved blazars underproduce the measured APS below $\sim 1 \mathrm{GeV}$. Contrary to past results, this suggests the presence of a new contribution to the lowenergy APS, with a significance of, at least, $5 \sigma$. The excess can be ascribed to a new class of faint gamma-ray emitters. If we consider the APS obtained by masking the sources in the 2FGL catalog, there is no underproduction of the APS below $1 \mathrm{GeV}$, but the new source class is still preferred over the blazars-only scenario (with a significance larger than $10 \sigma$ ). The properties of the new source class and the level of anisotropies induced in the isotropic gamma-ray background are the same, independent of the APS data used. In particular, the new gamma-ray emitters must have a soft energy spectrum, with a spectral index ranging, approximately, from 2.7 to 3.2. This complicates their interpretation in terms of known sources, since, normally, star-forming and radio galaxies are observed with a harder spectrum. The new source class identified here is also expected to contribute significantly to the intensity of the isotropic gamma-ray background.
\end{abstract}

DOI: $10.1103 /$ PhysRevD.95.123006

\section{INTRODUCTION}

In more than eight years of operation, the Fermi Large Area Telescope (LAT) has significantly increased the census of known gamma-ray emitters: the most recent source catalog (i.e. the Fermi LAT Third Source Catalogue, 3FGL) contains 3033 objects, detected with a significance greater than $4 \sigma$ between $100 \mathrm{MeV}$ and $300 \mathrm{GeV}$ [1]. Gamma-ray sources that are too faint to be resolved individually by Fermi LAT contribute cumulatively to the so-called isotropic gamma-ray background (IGRB). See Ref. [2] for a recent review.

The most recent measurement of the intensity energy spectrum of the IGRB was performed by Fermi LAT, and it covers the energy range between 0.1 and $820 \mathrm{GeV}$ [3]. By modeling known classes of gamma-ray emitters, Ref. [4] showed that the measured energy spectrum of the IGRB can be explained by the concomitant emission of unresolved blazars, star-forming and radio galaxies. However, the exact composition of the IGRB is still unknown: reconstructing it would provide valuable insight on the characteristics of the contributing source classes.

Different populations of gamma-ray emitters are expected to induce different levels of anisotropies in the

\footnotetext{
s.ando@uva.nl

†ornasam@gmail.com
}

IGRB (see Refs. [5-12] among others). Thus, a measurement of the gamma-ray angular power spectrum (APS) can constrain the nature of the IGRB in a complementary way with respect to the intensity energy spectrum. Other observables that can be employed to a similar goal are the 1-point photon count probability distribution of the IGRB [13-17], the cross correlation of the IGRB with catalogs of resolved galaxies [18-25] or of galaxy clusters [26], the cross-correlation with the weak gravitational lensing of cosmic shear [27-31] or with the gravitational lensing of the cosmic microwave background [32].

The first detection of the IGRB anisotropy APS was reported by the Fermi LAT Collaboration in 2012, in the energy range between 1 and $50 \mathrm{GeV}$ [33]. The signal was compatible with being due entirely to unresolved blazars $[9,10,12]$.

An updated measurement of the anisotropy APS has been recently released, employing 81 months of Fermi LAT data, binned in 13 energy bins, from 0.5 to $500 \mathrm{GeV}$ [34]. Apart from the autocorrelation APS in each energy bin, the new analysis measured, for the first time, the cross-correlation APS between different energy bins. Reference [34] suggested that the new APS data are due to more than one population of sources.

In this paper, we interpret the auto- and cross-correlation APS measured in Ref. [34] in terms of unresolved blazars. We employ a parametric model that was designed to 
describe the blazars observed by Fermi LAT [4,35-37]. The result of our fit to the new APS measurement will determine whether the latter can still be explained in terms of blazars alone or if an additional population of gammaray emitters is needed. Our analysis will also quantify the impact of the new APS measurement in constraining the properties of the blazar population, e.g., its redshift evolution and distribution in luminosity (especially at low luminosities). In general, this is the first work that takes advantage of the new anisotropy measurement to constrain the nature of the IGRB, employing physically motivated models of astrophysical emitters.

The paper is organized as follows: in Sec. II, we summarize the model used to describe the blazar population. We also discuss how to compute the observables in which we are interested, i.e. the anisotropy APS of unresolved blazars, the source count distribution function of resolved blazars and the intensity energy spectrum of all blazars (both resolved and unresolved). In Sec. III, we present the data employed in the fit and we describe our fitting technique. The results are presented in Sec. IV, while Sec. V and Sec. VI are left for the discussion and the conclusions, respectively.

\section{MODELING THE BLAZAR POPULATION}

The "blazar sequence" model $[38,39]$ is a multiwavelength description of blazars. It aims at reproducing the spectral energy distribution of blazars from radio to the $\mathrm{GeV}$ range. It also relates the shape of the spectral energy distribution to the luminosity of the source and, thus, it predicts a correlation between the x-ray and gamma-ray luminosities of the source. This correlation suggests that the properties of blazars in the gamma-ray range (e.g. their gamma-ray luminosity function and redshift evolution) should mimic what is observed in $\mathrm{x}$ rays. In particular, using data from ROSAT, Chandra and XMM-Newton, Refs. [40,41] showed that the X-ray luminosity function of $\mathrm{x}$-ray-selected active galactic nuclei is well fitted by a double power law in luminosity and that their redshift evolution is maximal at a certain "redshift peak," possibly as a result of the interplay between the growth of the central supermassive black hole over cosmic time and the falling off of fueling activity at late times. Refs. [40,41] also found evidence that such a peak depends on the source luminosity, i.e. the luminosity-dependent density evolution (LDDE).

The model defined in Refs. [4,36,37] to describe blazars in the gamma-ray regime is inspired by those findings and it reproduces quite well the blazars observed by the Fermi LAT.. ${ }^{1}$ For this reason, we decide to adopt it in the present work. We summarize its main features in the following.

The gamma-ray luminosity function of blazars, $\Phi\left(L_{\gamma}, z, \Gamma\right)$, is defined as the number of sources per unit

\footnotetext{
${ }^{1}$ Other works that followed a similar approach are Refs. [5,6, 10,42-44].
}

luminosity $L_{\gamma}$ (defined in the rest frame of the source, for energies between 0.1 and $100 \mathrm{GeV}$ ), per unit comoving volume $^{2} d V$ and per unit spectral photon index $\Gamma$. The luminosity function at redshift $z=0$ is modeled as a double power law in $L_{\gamma}$, as follows,

$$
\begin{aligned}
\Phi\left(L_{\gamma}, z=0, \Gamma\right)= & \frac{d N}{d L_{\gamma} d V d \Gamma} \\
= & \frac{A}{\ln (10) L_{\gamma}}\left[\left(\frac{L_{\gamma}}{L_{0}}\right)^{\gamma_{1}}+\left(\frac{L_{\gamma}}{L_{0}}\right)^{\gamma_{2}}\right]^{-1} \\
& \times \exp \left[-\frac{\left(\Gamma-\mu\left(L_{\gamma}\right)\right)^{2}}{2 \sigma^{2}}\right],
\end{aligned}
$$

where $\gamma_{1}$ and $\gamma_{2}$ are the indexes of the power laws and $L_{0}$ controls the transition between the two regimes. The factor $A$ sets the overall normalization and the exponential term describes a Gaussian distribution for the photon index $\Gamma$, with $\mu\left(L_{\gamma}\right)$ and $\sigma$ its mean and width, respectively. ${ }^{3}$ In the literature, blazars are often divided into two subclasses, flat-spectrum radio quasars (FSRQs) and BL Lacertae objects (BL Lacs). The two subclasses populate different regimes of the same correlation between luminosity and spectral index: FSRQs are brighter with a softer spectrum but, as the luminosity decreases, sources become harder and it is more common to find BL Lacs than FSRQs [46]. Allowing the mean $\mu\left(L_{\gamma}\right)$ of the spectral-index distribution to depend on $L_{\gamma}$, one can reproduce the $L_{\gamma}-\Gamma$ correlation and, thus, describe both FSRQs and BL Lacs within the same model. In particular, we assume $\mu\left(L_{\gamma}\right)$ to be parametrized as follows $[4,47,48]$ :

$$
\mu\left(L_{\gamma}\right)=\mu^{*}+\beta\left[\log \left(\frac{L_{\gamma}}{\operatorname{erg~s}^{-1}}\right)-46\right] .
$$

The values of the constant $\mu^{*}$ and $\beta$ are discussed in Sec. III. The redshift evolution of the gamma-ray luminosity function is described by the evolutionary factor $e\left(z, L_{\gamma}\right)[4,36]$,

$$
\Phi\left(L_{\gamma}, z, \Gamma\right)=\Phi\left(L_{\gamma}, z=0, \Gamma\right) e\left(z, L_{\gamma}\right),
$$

with

$e\left(z, L_{\gamma}\right)=\left[\left(\frac{1+z}{1+z_{c}\left(L_{\gamma}\right)}\right)^{-p_{1}\left(L_{\gamma}\right)}+\left(\frac{1+z}{1+z_{c}\left(L_{\gamma}\right)}\right)^{-p_{2}\left(L_{\gamma}\right)}\right]^{-1}$.

\footnotetext{
${ }^{2}$ We employ cosmological parameters in agreement with the observations by Planck [45].

${ }^{3}$ There is no a priori reason to assume that the spectral index $\Gamma$ follows a Gaussian distribution. However, it provides a good description for the blazars detected by Fermi LAT. See top left panel of Fig. 1 in Ref. [4].
} 
The critical redshift $z_{c}$ determines the epoch where the evolution changes behavior and it is inspired by the "redshift peak" found for x-ray-selected active galactic nuclei. The indexes $p_{1}\left(L_{\gamma}\right)$ and $p_{2}\left(L_{\gamma}\right)$ control the redshift evolution below and above $z_{c}$. A positive (negative) index corresponds to a positive (negative) evolution, i.e. the gamma-ray luminosity function increasing (decreasing) with $z$. According to the LDDE evolutionary scheme, the critical redshift depends on the luminosity as follows:

$$
z_{c}\left(L_{\gamma}\right)=z_{c}^{*}\left(\frac{L_{\gamma}}{10^{48} \operatorname{erg~s}^{-1}}\right)^{\alpha}
$$

where $\alpha$ is constant (see Sec. III).

In Ref. [4] the indexes $p_{1}$ and $p_{2}$ are also allowed to depend linearly on the logarithm of the luminosity, as follows:

$$
\begin{aligned}
& p_{1}\left(L_{\gamma}\right)=p_{1}^{0}+\tau\left[\log \left(\frac{L_{\gamma}}{\operatorname{erg~s}^{-1}}\right)-44\right], \\
& p_{2}\left(L_{\gamma}\right)=p_{2}^{0}+\delta\left[\log \left(\frac{L_{\gamma}}{\operatorname{erg~s}^{-1}}\right)-44\right] .
\end{aligned}
$$

$p_{1}^{0}$ and $p_{2}^{0}$ control the $L_{\gamma}$-independent parts of $p_{1}$ and $p_{2}$, respectively, and $\tau$ and $\delta$ their linear dependence. Their values will be discussed in Sec. III.

Other evolutionary scenarios, with different $e\left(z, L_{\gamma}\right)$ and modified versions of Eq. (3) have been considered in the literature. Ref. [4] employs them to describe a sample of 403 blazars detected by Fermi LAT with a test statistic larger than 50, at Galactic latitudes $|b|$ larger than $15^{\circ}$ [49]. Even if the likelihood analysis of Ref. [4] is not able to significantly distinguish between the evolutionary schemes considered, the LDDE is the one yielding the largest loglikelihood. Thus, in this work, we restrict our analysis to the LDDE scheme.

Blazars are best described by a curved energy spectrum. Thus, we model their energy spectrum $d N_{\gamma} / d E$ as follows:

$$
\frac{d N_{\gamma}}{d E_{\gamma}} \propto\left[\left(\frac{E_{\gamma}}{E_{b}}\right)^{\gamma_{a}}+\left(\frac{E_{\gamma}}{E_{b}}\right)^{\gamma_{b}}\right]^{-1} \exp \left[-\tau_{\mathrm{EBL}}\left(E_{\gamma}, z\right)\right]
$$

where $E_{\gamma}$ is the observed gamma-ray energy. We assume that $E_{b}$ correlates with $\Gamma$ according to $\log \left(E_{b} / \mathrm{GeV}\right)=$ $9.25-4.11 \Gamma[4]$. The factor $\exp \left[-\tau_{\mathrm{EBL}}\left(E_{\gamma}, z\right)\right]$ accounts for the absorption of gamma rays due to their interaction with the extragalactic background light through pair conversion. We model it following Ref. [50].

According to the model defined above, the differential source count distribution of blazars $d N / d F$ (i.e. the number of sources per unit solid angle and per unit flux, measured in $\mathrm{cm}^{2} \mathrm{~s} \mathrm{deg}^{-2}$ ) can be written as follows, $\frac{d N}{d F}=\int_{0.01}^{5.0} d z \int_{1}^{3.5} d \Gamma \Phi\left[L_{\gamma}\left(F_{\mathrm{E}}, z, \Gamma\right), z, \Gamma\right] \frac{d V}{d z} \frac{d L_{\gamma}}{d F}$,

where $F$ denotes the number flux above $100 \mathrm{MeV}$, as opposed to the energy flux $F_{\mathrm{E}}$. The quantity $L_{\gamma}\left(F_{\mathrm{E}}, z, \Gamma\right)$ is the luminosity associated with a source with flux $F_{\mathrm{E}}$ at a redshift $z$ and with spectral index $\Gamma$. The bounds of the integration in $\Gamma$ in Eq. (9) are chosen to properly sample the distribution of $\Gamma$, while those in redshift probe the region where the majority of the emission comes from. In particular, we assume that there are no blazars below $z=0.01[4]$.

The cumulative intensity energy spectrum $d I / d E_{\gamma}$ of all blazars (i.e. resolved and unresolved) can be computed (in units of $\mathrm{cm}^{-2} \mathrm{~s}^{-1} \mathrm{sr}^{-1} \mathrm{GeV}^{-1}$ ) as follows:

$$
\begin{aligned}
\frac{d I}{d E_{\gamma}}= & \int_{0.01}^{5.0} d z \frac{d V}{d z} \int_{1}^{3.5} d \Gamma \int_{L_{\min }}^{L_{\max }} d L_{\gamma} \Phi\left(L_{\gamma}, z, \Gamma\right) \\
& \times F\left(L_{\gamma}, z, \Gamma\right) \frac{d N_{\gamma}\left(\Gamma, z, E_{\gamma}\right)}{d E_{\gamma}} .
\end{aligned}
$$

Similarly to Eq. (9), $F\left(L_{\gamma}, z, \Gamma\right)$ is the flux (between 0.1 and $100 \mathrm{GeV}$ ) produced by a source with luminosity $L_{\gamma}$, spectral index $\Gamma$ and at a redshift $z$, and $d N_{\gamma} / d E_{\gamma}$ is the energy spectrum from Eq. (8), properly normalized so that $F d N_{\gamma} / d E$ provides the differential flux of the source. The upper bound in the integration in $L_{\gamma}$ is fixed at $10^{52} \mathrm{erg} \mathrm{s}^{-1}$ and the lower one at $10^{43} \mathrm{erg} \mathrm{s}^{-1}$. Their precise values are not particularly important as the integrand in Eq. (10) drops quickly at low and high luminosities.

Finally, for the APS $C_{\mathrm{P}}^{i, j}$ between energy bins $i$ and $j$ ( $i=j$ for the auto-correlation APS and otherwise for the cross-correlation), we assume that blazars are pointlike and that their APS is dominated by their so-called 1-halo term [5,51]. This is a good assumption if the sources producing the anisotropy signal are relatively bright and not numerous, which is the case for unresolved blazars $[5,6]$. In that case, the APS is Poissonian, i.e. independent of angular multipoles. It can be computed as follows (in units of $\left.\mathrm{cm}^{-4} \mathrm{~s}^{-2} \mathrm{sr}^{-1}\right)$ :

$$
\begin{aligned}
C_{\mathrm{P}}^{i, j}= & \int_{0.01}^{5.0} d z \frac{d V}{d z} \int_{1}^{3.5} d \Gamma \int_{L_{\min }}^{L_{\max }} d L_{\gamma} \Phi\left(L_{\gamma}, z, \Gamma\right) \\
& \times F_{i}\left(L_{\gamma}, z, \Gamma\right) F_{j}\left(L_{\gamma}, z, \Gamma\right) \\
& \times\left[1-\Omega\left(F_{\mathrm{E}}\left(L_{\gamma}, z, \Gamma\right), \Gamma\right)\right] .
\end{aligned}
$$

The quantity $\Omega(F, \Gamma)$ is the "sky coverage" and it describes the probability of Fermi LAT to resolve a source characterized by $(F, \Gamma)$. It accounts for the fact that the telescope has a lower (i.e. better) sensitivity for harder sources. Note that the number fluxes $F_{i}$ and $F_{j}$ are integrated inside energy bins $i$ and $j$, respectively, while the energy flux $F_{E}$ in the sky coverage is integrated 
ANDO, FORNASA, FORNENGO, REGIS, and ZECHLIN

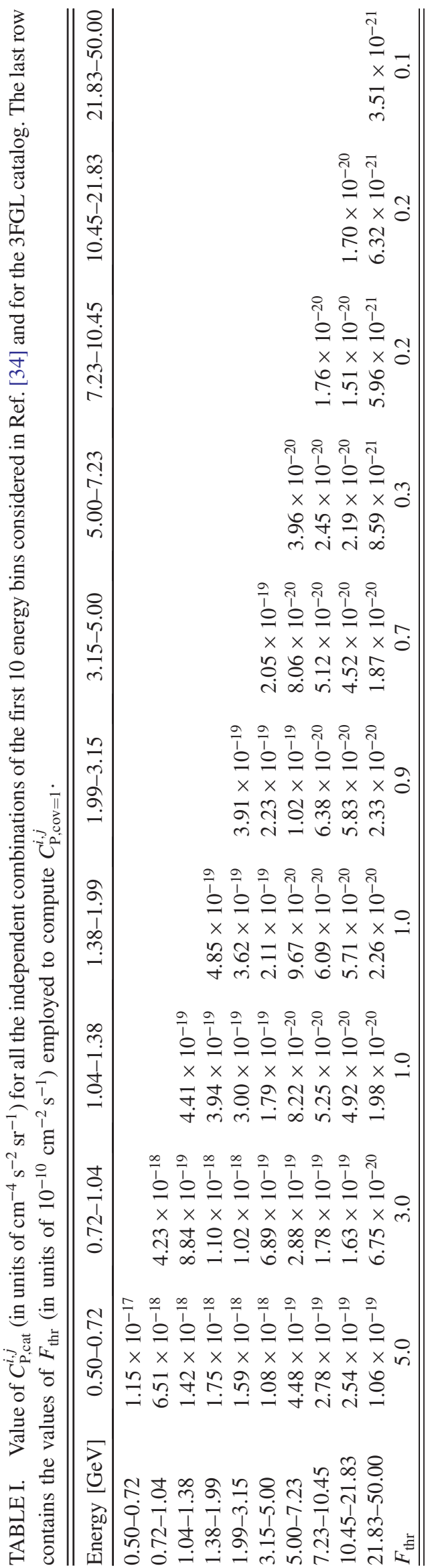

PHYSICAL REVIEW D 95, 123006 (2017)

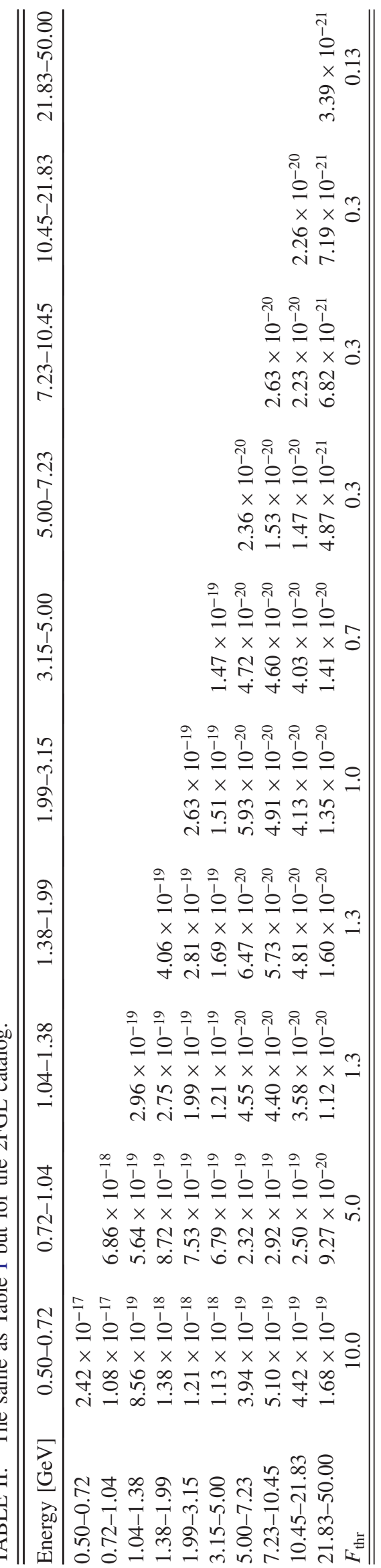


between 0.1 and $100 \mathrm{GeV}$. However, we ignore the exact behavior of $\Omega$ for faint sources. Also, note that the estimated $C_{\mathrm{P}}^{i, j}$ is very sensitive to how one models the transition between resolved and unresolved sources. Therefore, we decide to follow the procedure adopted in Ref. [17] and we compute $C_{\mathrm{P}}^{i, j}$ as the difference of two terms, i.e. $C_{\mathrm{P}}^{i, j}=C_{\mathrm{P}, \mathrm{cov}=1}^{i, j}-C_{\mathrm{P}, \mathrm{cat}}^{i, j}$. Here, $C_{\mathrm{P}, \mathrm{cov}=1}^{i, j}$ is the APS produced by all sources up to a certain threshold, $F_{\text {thr }}$, large enough that $\Omega\left(F_{\text {thr }}, \Gamma\right)=1$ for all values of $\Gamma$. This threshold depends on the energy bin considered and $C_{\mathrm{P}, \text { cov }=1}^{i, j}$ is computed as in Eq. (11) but replacing the factor $(1-\Omega)$ with $\Theta\left(F_{\text {thr }}^{i}-F^{i}\right) \Theta\left(F_{\text {thr }}^{j}-F^{j}\right)$, where $\Theta(x)$ is the Heaviside step function. The second term $C_{\mathrm{P}, \text { cat }}^{i, j}$ is the APS of the sources resolved in the catalog. It is computed directly from the catalog for all sources fainter than $F_{\text {thr }}$ and located at $|b|>30^{\circ}$. The fluxes $F^{i}$ and $F^{j}$ employed to compute $C_{\mathrm{P}, \text { cat }}^{i, j}$ are obtained by integrating the best-fit spectral model of each source, as provided in the catalog. For the Fermi LAT Second Source Catalogue (2FGL) [52] and 3FGL catalogs, it is known that those fits are not reliable for energies larger than few tens of $\mathrm{GeV}$ and, therefore, we only consider the APS $C_{\mathrm{P}}^{i, j}$ for energies below $50 \mathrm{GeV}$. By subtracting the contribution of resolved blazars, our method explicitly derives the APS of the unresolved ones without any assumptions on the sky coverage. In Ref. [17], it was tested that this procedure does not depend on the exact value chosen for $F_{\text {thr }}$, as long as it lies in a region close to the threshold and with coverage equal to 1.

Table I shows the values of $C_{\mathrm{P}, \text { cat }}^{i, j}$, for all the independent combinations of the first 10 energy bins considered in Ref. [34] and in the case of the 3FGL catalog. The last row shows the values of $F_{\text {thr }}$ employed for the different energy bins. Table II contains the same information but for the 2FGL catalog.

To conclude, we note that, contrary to $C_{\mathrm{P}}^{i, j}$, the way we compute the source count distribution in Eq. (9) and the intensity energy spectrum in Eq. (10) is independent of the telescope sensitivity, and it is not associated with a specific analysis or source catalog.

\section{DATA AND FITTING TECHNIQUE}

The auto- and cross-correlation APS are taken from Ref. [34]. The APS is computed from flux sky maps obtained after subtracting a model for the Galactic foreground, i.e. the emission induced by the interaction of cosmic rays with the interstellar medium and radiation fields. Also, the regions of the sky where resolved sources and the Galactic foreground are largely dominant are masked. Two masks are considered separately in Ref. [34]: they both mask the Galactic plane (i.e. the region with $|b|<30^{\circ}$ ) and few extended sources. Then, the "3FGL mask" excludes the region around each sources in the 3FGL catalog, while the "2FGL mask" removes all objects in the 2FGL catalog. Inside the multipole range considered in Ref. [34] (i.e. between $\ell=49$ and 706), both the auto- and cross-correlation APS are found compatible with being Poissonian. We only consider the best-fit APS $C_{\mathrm{P}}^{i, j}$ for the first 10 energy bins, i.e. below $50 \mathrm{GeV}$. The 55 independent $C_{\mathrm{P}}^{i, j}$ are taken from Tables I and II of Ref. [34].

The data on the source count distribution, in the case of the Fermi LAT First Source catalog (1FGL), are taken from Fig. 14 of Ref. [35] (data set labeled "all blazars") and they refer to emitters associated with blazars detected in the energy range between $100 \mathrm{MeV}$ and $100 \mathrm{GeV}$. Note that these data have already been corrected for the sky coverage, so that we can directly compare them with the model prediction computed in Eq. (9). For the 2FGL and 3FGL catalog, the $d N / d F$ is computed directly from the catalog as discussed in Appendix B of Ref. [15]. ${ }^{4}$ We restrict ourselves to a flux regime in which the catalogs are complete, i.e. above $1.98 \times 10^{-8} \mathrm{~cm}^{-2} \mathrm{~s}^{-1}$ for $2 \mathrm{FGL}$ and above $1.34 \times 10^{-8} \mathrm{~cm}^{-2} \mathrm{~s}^{-1}$ for 3FGL. ${ }^{5}$

Finally, we will also consider the intensity energy spectrum of the extragalactic gamma-ray background (EGB), i.e. the residual emission observed by Fermi LAT after subtracting a model of the Galactic foreground [3]. The EGB is interpreted as the emission of all sources (both resolved and unresolved), as opposed to the IGRB that only includes unresolved ones. The measurement in Ref. [3] is based on 50 months of data and it covers the energy range between $100 \mathrm{MeV}$ and $820 \mathrm{GeV}$. We consider the entries from Table 3 of Ref. [3], in the case of its "model A" for the Galactic foreground. Note that, in the following, we will not include the data of the EGB intensity energy spectrum in our fits. However, we will compare them to our prediction for the total emission associated with blazars.

The fits are performed by scanning over the parameters defining the blazar population and computing the likelihood function. Scans are performed with PyMultiNest ${ }^{6}$ [53], based on MultiNest v3.10 ${ }^{7}$ [54-56]. The tolerance is fixed at 0.5 with 5000 live points. This guarantees a good sampling of the likelihood so that results of the scans can be interpreted both in a Bayesian and frequentist framework.

We assume that all the data are independent ${ }^{8}$ and that they arise from a Gaussian probability distribution. Thus,

\footnotetext{
${ }^{4}$ Here, we consider sources detected with a significance larger than $6 \sigma$, at Galactic latitudes $|b|>30^{\circ}$ and associated with blazars, i.e. classes $\mathrm{bzb}, \mathrm{bzq}$ and agu for the 2FGL catalog and classes bll, fsrg and bcu for the 3FGL one.

${ }^{5}$ Both fluxes are computed above $100 \mathrm{MeV}$.

${ }^{6} \mathrm{http}: / /$ johannesbuchner.github.io/PyMultiNest/index.html.

${ }^{7}$ https://ccpforge.cse.rl.ac.uk/gf/project/multinest/.

${ }^{8}$ As discussed in Ref. [34], the covariances between two measured $C_{\mathrm{P}}^{i, j}$ are negligible. Also, we decide to work with the differential $d N / d F$, instead of the cumulative one $N(>F)$, so that we can neglect the covariance between the source count distribution in different flux bins
} 
the logarithm of the likelihood function $\ln \mathcal{L}(\boldsymbol{\Theta})$ is proportional to $-\sum_{i}\left[\bar{D}_{i}-D_{i}(\boldsymbol{\Theta})\right]^{2} / 2 \bar{\sigma}_{i}^{2}$, where the sum runs over all the data considered. $\bar{D}_{i}$ is the measured value for data point $i$ and $\bar{\sigma}_{i}$ its estimated error, while $D_{i}(\boldsymbol{\Theta})$ is the value of the same observable estimated for point $\boldsymbol{\Theta}$ in the parameter space. Different scans will feature different parameter spaces and different data sets.

We start by considering a parameter space that is fourdimensional, comprised of parameters $A, \gamma_{1}, L_{0}$, and $p_{2}^{0}$ defined in Eqs. (1) and (7). The choice is made in order to guarantee a significant freedom and variability for the theoretical predictions, without having to deal with too many free parameters. In particular, $\gamma_{1}$ controls the gammaray luminosity function in the low-luminosity regime, while $A$ and $L_{0}$ control its overall normalization. The parameter $p_{2}^{0}$ determines the evolution of blazars with redshift. All the other parameters in Eqs. (1), (2), (5)-(8) are fixed to their median values from Ref. [4] (in the case of the LDDE evolutionary scheme). In particular, $\gamma_{2}=1.83$, $\mu^{*}=2.22, \quad \beta=0.10, \quad \sigma=0.28, \quad z_{c}^{*}=1.25, \quad \alpha=7.23$, $p_{1}^{0}=3.39, \tau=3.16, \delta=0.64, \gamma_{a}=1.7$ and $\gamma_{b}=2.6$. We implement $\log$ priors on $A$ and $L_{0}$ (between $10^{-6}$ and $10^{2} \mathrm{Gpc}^{-3}$ and between $10^{45}$ and $10^{53} \mathrm{erg} \mathrm{s}^{-1}$, respectively) and linear priors on $\gamma_{1}$ and $p_{1}^{0}$ (between 0 and 5, and between -20 and 0 , respectively). Different parameter spaces and prior distributions will be defined in the following sections.

For all the parameters $\Theta_{i}$ we compute the marginalized probability distribution function and the Profile Likelihood (PL). The former is obtained by integrating the posterior probability distribution over all parameters except $\Theta_{i}$, while the PL is computed by maximizing over them $[57,58]$. We also derive two-dimensional marginalized posterior distribution functions and PL for certain combinations of parameters. In all the cases considered, the frequentist (i.e. maximization of the likelihood) and the Bayesian (i.e. marginalization of the posterior distribution) approach yield similiar results. Thus, in the following sections, we show only the frequentist case.

\section{RESULTS}

\section{A. Fitting only the auto- and cross-correlation angular power spectrum (3FGL mask)}

We start by performing a scan over $A, \gamma_{1}, L_{0}$ and $p_{2}^{0}$, i.e. our model only includes blazars. The data set employed in the likelihood only includes the auto- and cross-correlation APS from Ref. [34] for the 3FGL mask. Thus, in this scan we try to reproduce the measured APS only in terms of blazars. The red regions in Fig. 1 show the two-dimensional PL for different combinations of the model parameters. The inner contours denote the $68 \%$ C.L. region and the outer ones the 95\% C.L. The black circles are the best fits. It appears that the reconstruction of the model parameters is still affected by significant degeneracies. For example, the upper middle panel of Fig. 1 shows a degeneracy between $A$ and $L_{0}$ since, for a fixed value of $\gamma_{1}$, they both control the normalization of the gamma-ray luminosity function, see Eq. (1). Also, from the upper left panel, increasing (decreasing) $\gamma_{1}$ corresponds to a lower (larger) $A$, since making the gamma-ray luminosity function steeper (shallower) at low fluxes increases (decreases) the total number of low-luminosity sources and, thus, a larger (smaller) normalization is needed to reproduce the measured APS. Another way of increasing the abundance of low-flux sources is to increase $p_{2}^{0}$ and, thus, shifting blazars to larger redshifts. This gives rise to the degeneracy in the upper panel.

Figure 2 shows the predicted auto-correlation APS (left panel) and the $3 \mathrm{FGL} d N / d F$ (right panel), in comparison with the data points used in the fit (gray boxes). The best-fit solution is represented by the full red circles and the solid red line, embedded in the $68 \%$ (red) and 95\% C.L. (pink) error bar. We note that, even if we do not include the measured source count distribution in our fit, the best-fit solution describes the data reasonably well (right panel of Fig. 2). On the other hand, the best fit underpredicts the auto-correlation APS in the first energy bin, but it is compatible with the measured APS at 95\% C.L. ${ }^{9}$ This discrepancy is responsible for the best-fit $\chi^{2}=-2 \ln \mathcal{L}$ of 80.88 , corresponding to a $\chi^{2}$ per degree of freedom of 1.47 and a $p$-value of 0.005 . Note that the best-fit solution predicts negligible anisotropies above $50 \mathrm{GeV}$. The drop of the red line above $50 \mathrm{GeV}$ is compatible with the fact that, at high energies, the measured APS has a detection significance lower than $3 \sigma$ [34].

From Fig. 1, we also note that both $\gamma_{1}$ and $p_{2}^{0}$ prefer values that are at the edge of the prior range considered. This suggests that increasing the range may lead to a solution with a better $\chi^{2}$. We do not consider the possibility of negative $\gamma_{1}$ since there is no indication of this in the analysis of resolved blazars from Ref. [4], but we perform another scan with a prior range for $p_{2}^{0}$ that extends up to 20.0. Even if a positive evolution of blazars for $z>z_{\mathrm{c}}$ is probably unphysical and against the findings of Ref. [4], we consider this possibility as it may increase the abundance of blazars at low energies and, thus, potentially improve the agreement with the APS below $1 \mathrm{GeV}$. However, the bestfit value for $p_{2}^{0}$ results to be $0.23_{-0.59}^{+1.72}$ and the best-fit APS still underproduces the low-energy APS.

\section{B. Adding the source count distribution (3FGL mask)}

A realistic model of blazars needs to reproduce the number of sources observed by Fermi LAT. Thus, we perform a new scan over the same four parameters as in the

\footnotetext{
${ }^{9}$ Similarly, the best-fit solution underestimates most of the cross-correlation data points involving the first two energy bins.
} 

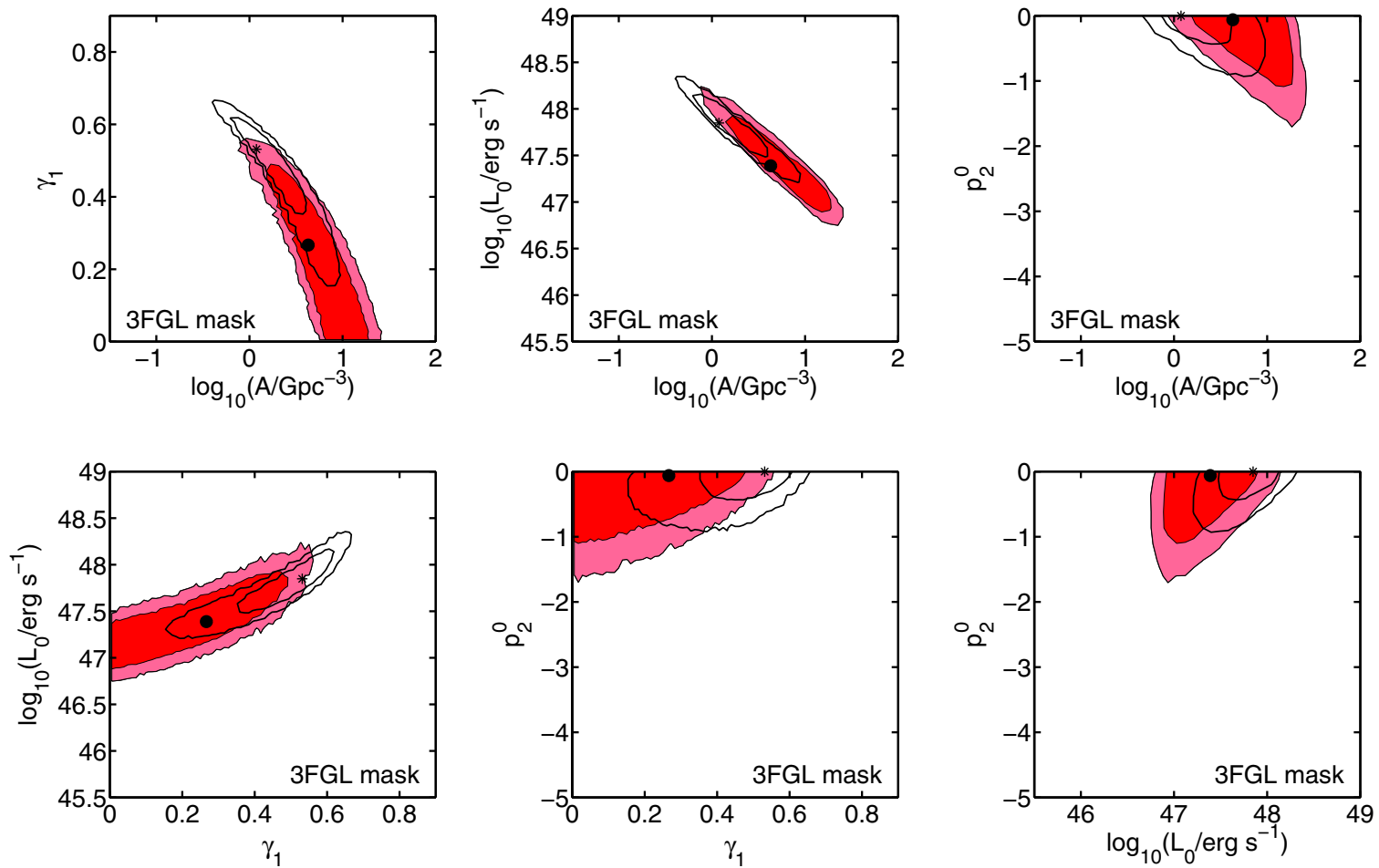

FIG. 1. Two-dimensional profile-likelihood contour plots for all possible combinations of model parameters $A, \gamma_{1}, L_{0}$ and $p_{2}^{0}$. The contours indicate the level of agreement between the model predictions and the data considered in the fit. The model only accounts for the contribution of blazars (no additional source class). The filled red contours and the black dots refer to a fit to the auto- and crosscorrelation APS from Ref. [34] (see Sec. IVA). In the case of the empty (transparent) areas and the black stars we fit also the source count distribution $d N / d F$ from the 3FGL catalog (see Sec. IV B). For each set of contours, the inner one indicates the $68 \%$ C.L. region and outer one the $95 \%$ C.L. region.

previous section, fitting, at the same time, the auto- and cross-APS from Ref. [34] (for the 3FGL mask) and the observed source count distribution $d N / d F$. This new scan attempts to employ only blazars to describe both the measured APS and the observed $d N / d F$. We only show results for the $d N / d F$ of the 3FGL catalog but we performed separate scans for the 1FGL and 2FGL $d N / d F$. Results are qualititatively similar to what is presented in the following.

The empty (transparent) areas in Fig. 1 show the twodimensional PL for all possible combinations of model parameters. The black stars show the best-fit point. The best-fit solution is not very different from the fit to the APS data alone (see Sec. IVA) and the preferred regions are located along the same degeneracies as before, with a slight shift towards smaller normalisations $A$ and, therefore, larger values of $\gamma_{1}$ and $L_{0}$. The PL distribution for $p_{2}^{0}$ is still clustered towards the upper edge of its prior range, while, this time, $\gamma_{1}$ is different from zero at $95 \%$ C.L. Solutions with a $\gamma_{1} \sim 0$ would underestimate the source count distribution and are thus excluded. In Fig. 3, we compare again the data used in the scan (gray boxes) to the best-fit model predictions (solid blue lines and blue squares). The dark blue (light blue) vertical bars around the squares denote the $68 \%$ and $95 \%$ C.L. uncertainty.
Similar to the previous section, we note an underestimation of the predicted autocorrelation APS below $1 \mathrm{GeV}$. The best-fit solution has a $\chi^{2}$ of 112.41 , corresponding to a $\chi^{2}$ per degree of freedom of 1.70 and a $p$-value of $3 \times 10^{-4}$. Allowing $p_{2}^{0}$ to have positive values leads to a best-fit $p_{2}^{0}$ that is different from zero at $95 \%$ C.L. (i.e. $p_{2}^{0}=2.48_{-0.73}^{+0.81}$ ). In that case, the best-fit solution has a $\chi^{2}$ value of 106.10 , but the reconstructed APS still underproduces the measured one below $1 \mathrm{GeV}$. The results of these first two scans suggest that blazars alone are not able to reproduce the measured APS below $\sim 1 \mathrm{GeV}$. This agrees with the findings of Ref. [34].

We also tested this result by performing a scan in which we fit the APS with the 3FGL mask and the 3FGL $d N / d F$ but, this time, the source count distribution is computed with all sources in the catalog, not only blazars. In this case, the best-fit solution predicts approximately $10 \%$ more APS below $1 \mathrm{GeV}$ and, thus, the best-fit $\chi^{2}$ is smaller (i.e. $\left.\chi^{2}=93.78\right)$ than with the blazars $d N / d F$. This suggests that taking unassociated sources into account could improve the agreement with the measured APS. We furher comment on that in Sec. V.

We note that our best-fit values from Fig. 1 are in agreement (at the 95\% C.L.) with the results of Ref. [4]. In that paper, the authors employ the same LDDE scheme 

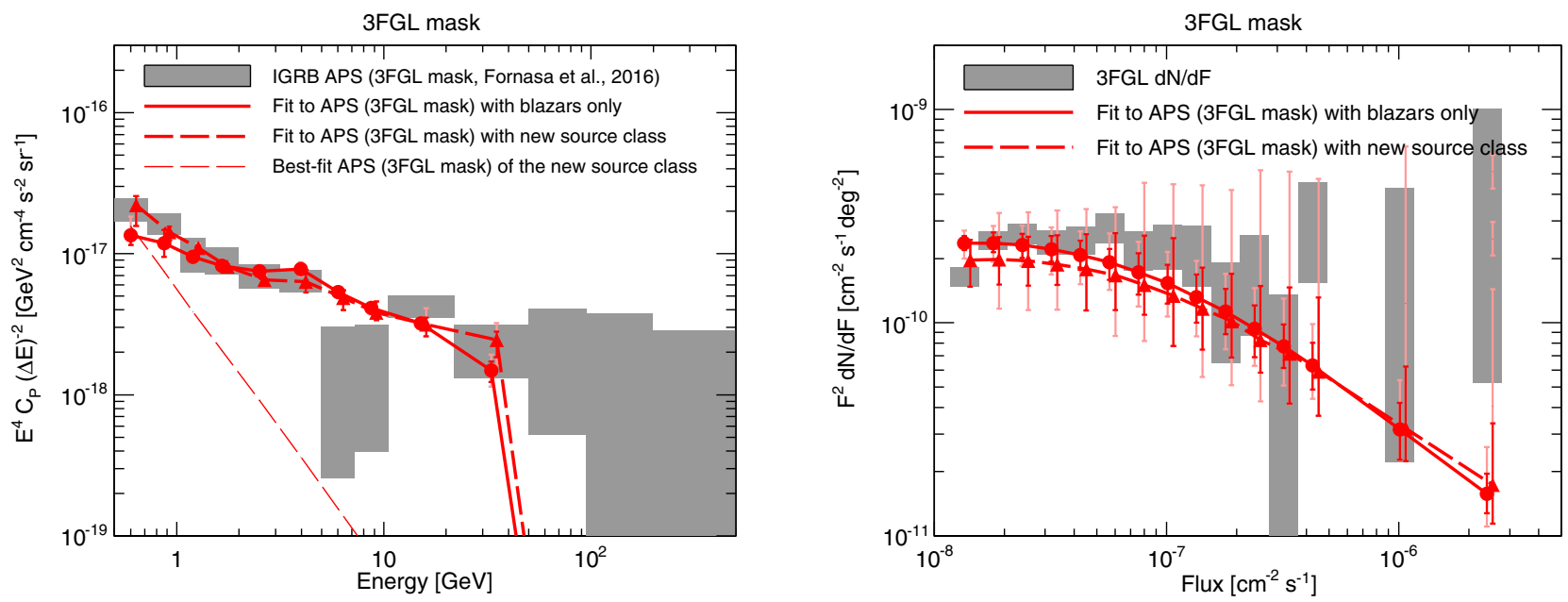

FIG. 2. The panels compare the results of our fits (red lines, circles and triangles) to different data sets (gray boxes). The left panel is for the auto-correlation APS with the 3FGL mask and the right panel is for the 3FGL $d N / d F$. For the fits considered here only the autoand cross-correlation APS from Ref. [34] are included in the likelihood. The thicker solid red lines and the red circles denote the best-fit solution for a model in which only blazars are included (no new source class, see Sec. IV A). The thicker dashed red line and the red triangles show the best-fit solution for a model including both blazars and the new source class (see Sec. IV C). The thinner red dashed line in the left panel shows the best-fit auto-correlation APS for the new class source. Vertical error bars indicate the $68 \%$ (in red) and 95\% C.L. (in pink) uncertainty. Circles and triangles are slightly shifted with respect to each other to increase readability.
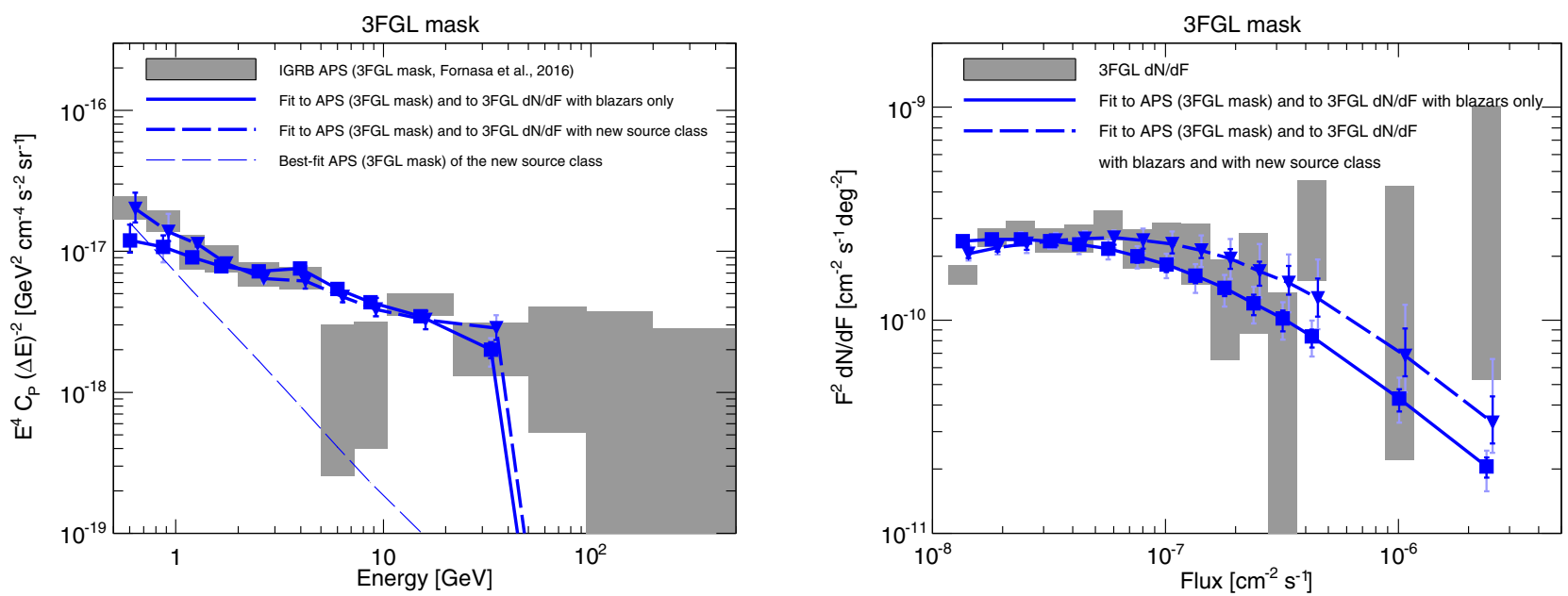

FIG. 3. The panels compare the results of our fits (blue lines, squares and triangles) to different data sets (gray boxes). The left panel is for the auto-correlation APS with the 3FGL mask and the right panel is for the 3FGL $d N / d F$. For the fits considered here the auto- and cross-correlation APS from Ref. [34] are included in the likelihood, as well as the 3FGL $d N / d F$. The thicker solid blue lines and the blue squares denote the best-fit solution for a model in which only blazars are included (no new source class, see Sec. IV B). The thicker dashed blue lines and the blue triangles show the best-fit solution for a model including both blazars and the new source class (see Sec. IV C). The thinner blue dashed line in the left panel shows the best-fit auto-correlation APS for the new class source. Vertical error bars indicate the $68 \%$ (in dark blue) and 95\% C.L. (in light cyan) uncertainty. Squares and triangles are slightly shifted with respect to each other to increase readability.

used here to describe 403 blazars observed by Fermi LAT, but they used a larger parameter space and they constrained their model by fitting the blazars' flux and spectral index distributions, also taking advantage of some redshift estimates.

\section{Including a new class of sources (3FGL mask)}

Motivated by the previous results, we expand our theoretical model by including an additional population of sources. We adopt a phenomenological description and we assume that the new sources emit power-law energy spectra $\left(\propto E^{-\Gamma_{\text {new }}}\right)$ and that they are pointlike and unclustered. Thus, their auto- and cross-correlation APS can be written as follows:

$$
C_{\mathrm{P}}^{i, j}=C_{\mathrm{P}}^{0,0} \frac{\left[E_{\mathrm{max}, i}^{1-\Gamma_{\text {new }}}-E_{\mathrm{min}, i}^{1-\Gamma_{\text {new }}}\right]\left[E_{\mathrm{max}, j}^{1-\Gamma_{\text {new }}}-E_{\mathrm{min}, j}^{1-\Gamma_{\text {new }}}\right]}{\left[E_{\mathrm{max}, 0}^{1-\Gamma_{\text {new }}}-E_{\mathrm{min}, 0}^{1-\Gamma_{\text {new }}}\right]^{2}},
$$



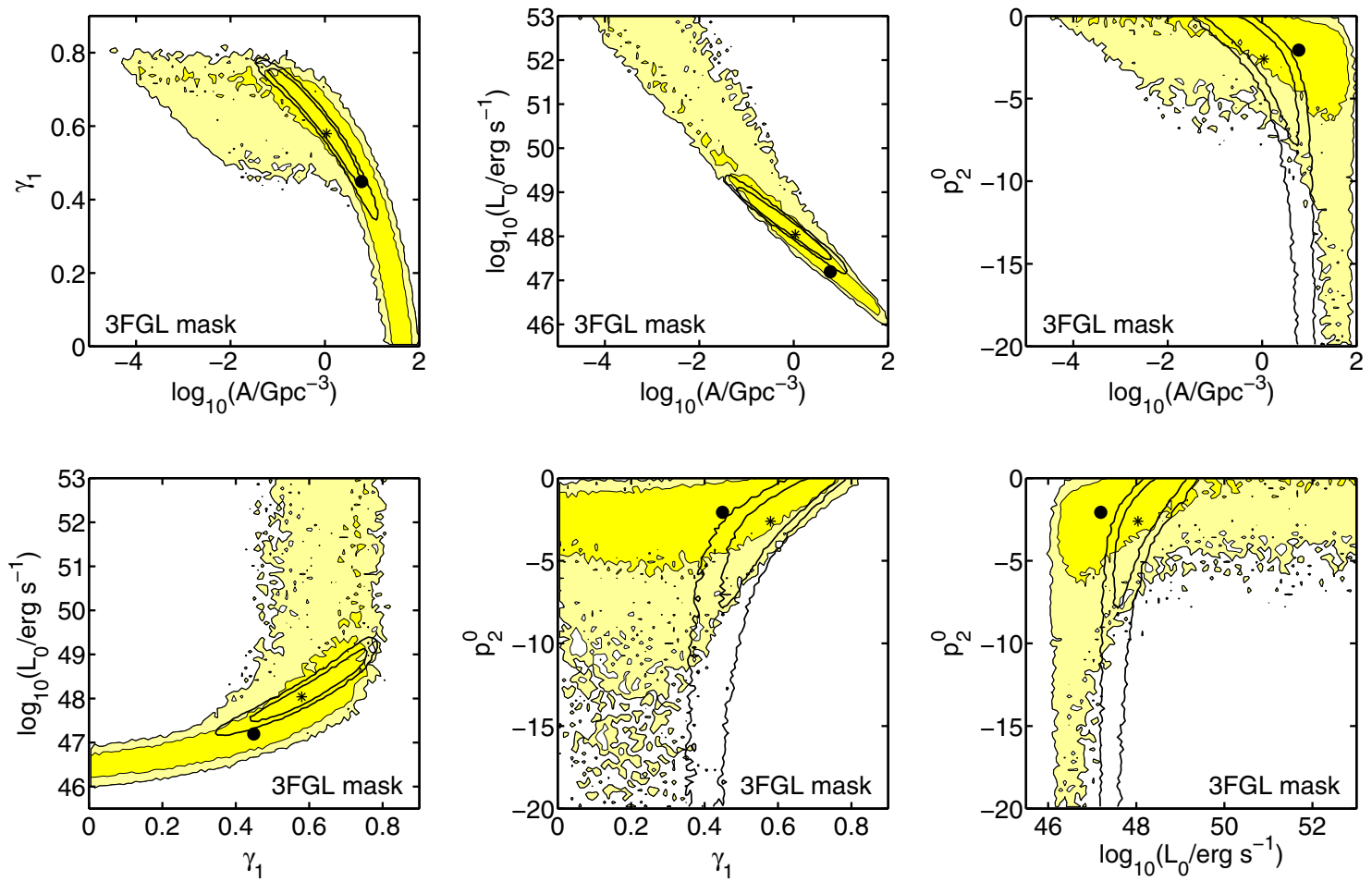

FIG. 4. Two-dimensional profile-likelihood contour plots for all possible combinations of model parameters $A, \gamma_{1}, L_{0}$ and $p_{2}^{0}$. The contours indicate the level of agreement between the model predictions and the data considered in the fit. The model includes the contribution of blazars and of the new source class (see Sec. IV C). The filled yellow contours and the black dots refer to a fit to the autoand cross-correlation APS from Ref. [34]. In the case of the empty (transparent) areas and the black stars we fit also the source count distribution $d N / d F$ from the 3FGL catalog. For each set of contours, the inner one indicates the $68 \%$ C.L. region and outer one the $95 \%$ C.L. region.

where $C_{\mathrm{P}}^{0,0}$ is the autocorrelation APS of the new population in the first energy bin, between $E_{\min , 0}$ and $E_{\max , 0}$, while $E_{\min , i}$ and $E_{\max , i}$ indicate the lower and upper bound of the $i$-th energy bin.

We perform one additional scan fitting the auto- and cross-correlation APS from Ref. [34] for the 3FGL mask but adding the contribution of the new source class in the computation of the model prediction. In this way we aim at reproducing the APS data by using two distinct source classes: blazars and a phenomenological speculative new population. $\Gamma_{\text {new }}$ and $C_{\mathrm{P}}^{0,0}$ are added to the list of free parameters in the scan. We assume flat priors for $\Gamma_{\text {new }}$ between 2.2 and 3.4 and $\log$ priors for $C_{\mathrm{P}}^{0,0}$ between $10^{-20}$ and $10^{-15} \mathrm{~cm}^{-4} \mathrm{~s}^{-2} \mathrm{sr}^{-1}$.

The two-dimensional $68 \%$ and $95 \%$ C.L. regions for all possible combinations of parameters $A, \gamma_{1}, L_{0}$ and $p_{2} 2^{0}$ are shown in Fig. 4 by the dark yellow (68\% C.L.) and light yellow (95\% C.L.) areas. The full black circles indicate the best fit. We note that, with respect to Fig. 1, the size of the contours has increased significantly: apart from the same degeneracy present in Fig. 1 (i.e. the diagonal band between $A$ and $\gamma_{1}$, for $A>1 \mathrm{Gpc}^{-3}$ ), a new region appears with $A<1 \mathrm{Gpc}^{-3}, \gamma_{1}>0.5$ and $L_{0}>10^{48} \mathrm{erg} \mathrm{s}^{-1}$. There is also another new region for large $A$ and very negative $p_{2}^{0}$. Models in both of these regions significantly underproduce the measured auto- and cross-APS at low energies, and therefore, they would be excluded if blazars were the only class of gamma-ray emitters in the fit.

The new source population takes care of improving the agreement with the low-energy APS. We can see this in Fig. 2, where the thick dashed red lines and the red triangles show the predicted best-fit autocorrelation APS (left panel) and 3FGL $d N / d F$ (right panel). As before, the vertical red (pink) lines indicate the 68\% (95\%) C.L. uncertainty. The autocorrelation APS of the new component alone is shown separately by the thinner dashed red line. The predicted $d N / d F$ (dashed red line in the right panel) is much more uncertain than with blazars only and it systematically underproduces the 3FGL source count distribution.

The yellow contours in Fig. 5 show the two-dimensional PL distributions for the new parameters in the scans, i.e. $C_{\mathrm{P}}^{0,0}$ and $\Gamma_{\text {new }}$. The PL of $C_{\mathrm{P}}^{0,0}$ is quite peaked and the reconstruction has a precision of $15 \%$. The reconstruction is less precise for $\Gamma_{\text {new }}$ and values between 2.79 and 3.27 are allowed at $68 \%$ C.L.

The best-fit $\chi^{2}$ is significantly better than without the new source class, i.e. $\chi^{2}=54.29$, corresponding to a $\chi^{2}$ per degree of freedom of 1.11 and a $p$-value of 0.28 . We can perform a likelihood-ratio test by defining $\Delta \chi^{2}$, i.e. the difference between the best-fit $\chi^{2}$ of the simpler model 


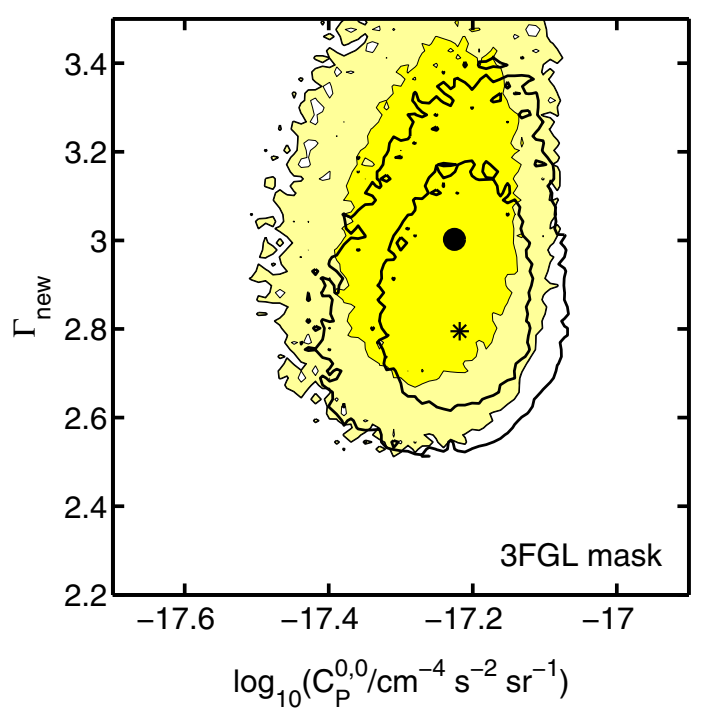

FIG. 5. Two-dimensional profile-likelihood contour plot for the parameters describing the new source class, i.e. $C_{\mathrm{P}}^{0.0}$ and $\Gamma_{\text {new }}$. The contours indicate the level of agreement between the model predictions and the data considered in the fit. The model includes the contribution of blazars and of the new source class (see Sec. IV C). The filled yellow contours and the black dots refer to a fit to the auto- and cross-correlation APS from Ref. [34]. In the case of the empty (transparent) areas and the black stars we fit also the source count distribution $d N / d F$ from the 3FGL catalog. For each set of contours, the inner one indicates the $68 \%$ C.L. region and outer one the $95 \%$ C.L. region.

(i.e. without the new source class, see Sec. IVA) and the best-fit $\chi^{2}$ of the one with the new source class. Since the simpler model is located on the boundary of the more complex one, we apply Chernoff's theorem $[59,60]$ and obtain a $p$-value of $5 \times 10^{-7}$, indicating that the model with the additional source class is preferred over the interpretation in terms of only blazars at $5 \sigma$.

Alternatively, following Bayesian statistics, the comparison between models can be performed by computing the Bayes factor $B$, defined as the ratio of the evidences of the two scans. In our scan $\ln B$ is 12.37 , a value suggesting a strong preference for the model with the new source class, according to Jeffrey's scale [61].

Finally, we perform one additional scan for which model predictions are computed in terms of blazars and the new source class but we also fit the $3 \mathrm{FGL} d N / d F$ (not only the APS). This scan employs blazars and the new source class to reproduce, at the same time, the APS data and the observed 3FGL $d N / d F$. The two-dimensional PL for parameters $A, \gamma_{1}, L_{0}$ and $p_{2}^{0}$ are denoted by empty (transparent) areas in Fig. 4. The most relevant difference with respect to the fit to the APS data only (filled yellow regions in Fig. 4; see Sec. IV B) is the fact that values of $\gamma_{1}$ smaller than 0.3 are disfavored at $95 \%$ C.L., as well as $A<10^{-2} \mathrm{Gpc}^{-3}$. Solutions in those regions would underpredict or overpredict the source count distribution, respectively. Figure 3 compares the autocorrelation APS and $d N / d F$ of the best-fit solution (dashed thick blue lines and blue triangles) to the data (grey boxes). As in Fig. 2, including the new source improves the agreement with the APS data below $1 \mathrm{GeV}$. The autocorrelation APS of the new class alone is shown by the thin blue dashed line in the left panel of Fig. 3. The two-dimensional PL of $C_{\mathrm{P}}^{0,0}$ and $\Gamma_{\text {new }}$ is plotted as empty areas in Fig. 5. Results are compatible with the yellow contours, i.e. relative to the fit to only the APS data. This confirms that the properties of the new source class do not change if we include the $d N / d F$ data. In this case, the best-fit $\chi^{2}$ is 70.19 , corresponding to $\chi^{2}$ per degree of freedom of 1.10 and a $p$-value of 0.28 . The likelihood-ratio test yields a $p$-value of $2 \times 10^{-10}$, corresponding to more than $10 \sigma$. The presence of the new source class is strongly favored also in a Bayesian framework as the Bayes factor $\ln B$ is 19.13 .

\section{Results for the 2FGL mask}

We repeat the scans discussed in the previous section but we now consider the auto- and cross-APS obtained with the 2FGL mask. As in Sec. IV A, we start by fitting only the auto- and cross-APS (for the 2FGL mask) in terms of blazars, i.e. no additional source class. The blue contours in Fig. 6 show the two-dimensional PL of the model parameters. The inner blue contours are for the $68 \%$ C.L. region and the outer ones for the $95 \%$ C.L. The black circles mark the best-fit point. The contours approximately overlap with the red regions in Fig. 1, confirming that the APS data point towards the same source population, independent of the catalog mask used. Indeed, the best-fit solutions obtained by using the two different masks (fitting only the APS and without additional source class) are in agreement with each other at $68 \%$ C.L. The main difference between Figs. 6 and 1 is the size of the contours: for the 2FGL mask, the measurement of the auto- and cross-APS is characterized by smaller error bars than for the 3FGL mask and, therefore, a smaller portion of the parameter space can fit the data. Figure 8 compares the APS data (left panel) and the 3 FGL $d N / d F$ (right panel) with the best-fit solution (solid red line and red circles). Differently than in Fig. 2, there is no underestimation of the APS at low energies. However, few discrepancies (especially for the data point around $1 \mathrm{GeV}$ and around $6 \mathrm{GeV}$ ) yield a best-fit $\chi^{2}$ of 117.24 , with a $\chi^{2}$ per degree of freedom of 2.30 , corresponding to a $p$-value of $4 \times 10^{-7} .^{10}$

The situation remains qualitatively unchanged when we include the fit to the 3FGL $d N / d F$. In that case, we aim at reproducing both the APS data (with the 2FGL mask) and the 3 FGL $d N / d F$ only in terms of blazars. The empty (transparent) areas in Fig. 6 show the resulting two-dimensional

\footnotetext{
${ }^{10}$ Allowing $p_{2}^{0}$ to be positive would improve the quality of the fit.
} 

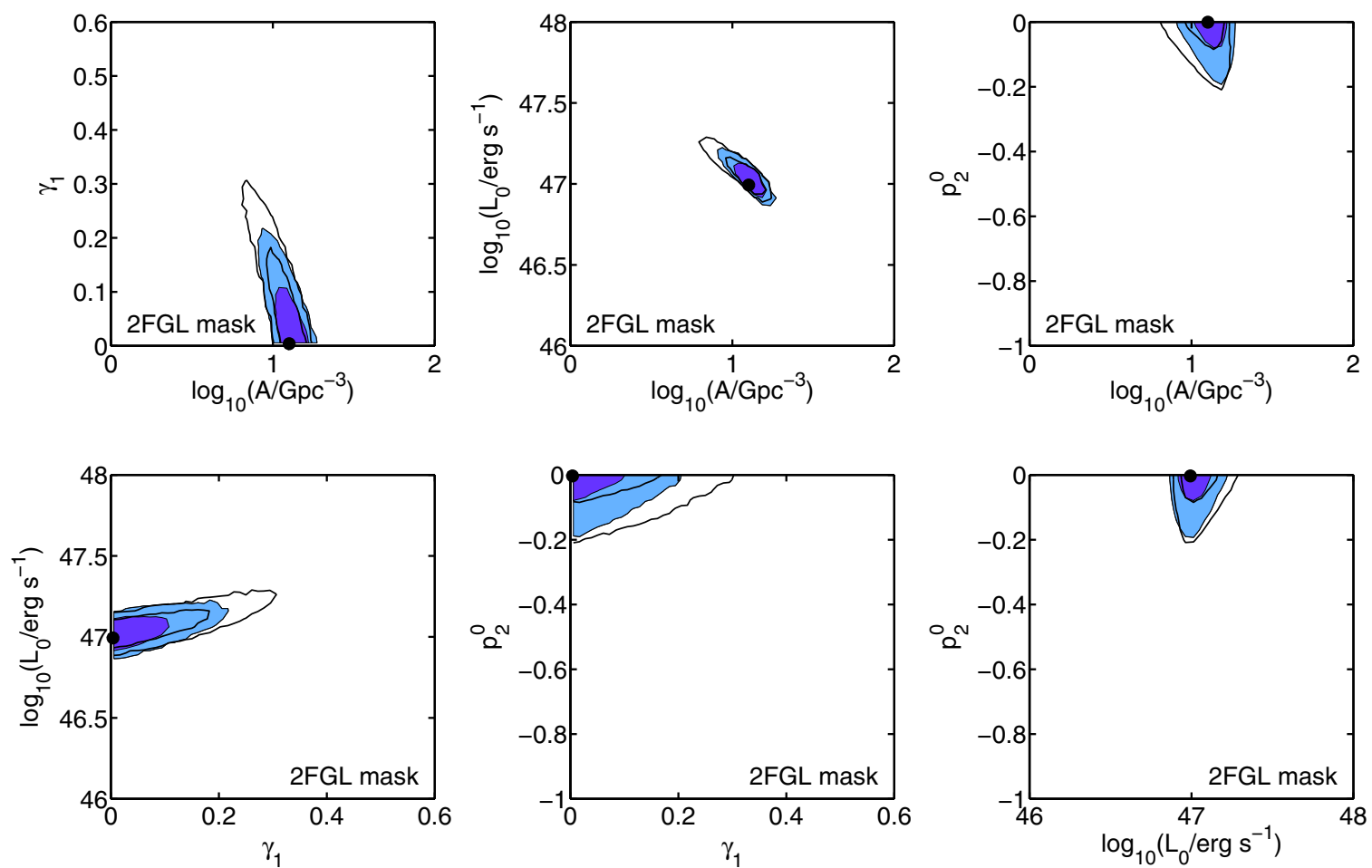

FIG. 6. Two-dimensional profile-likelihood contour plot for all the combinations of parameters $A, \gamma_{1}, L_{0}$ and $p_{2}^{0}$. The contours indicate the level of agreement between the model predictions and the data considered in the fit. The model only accounts for the contribution of blazars (no additional source class), see Sec. IV D. The filled blue contours and the black dots refer to a fit to the auto- and crosscorrelation APS from Ref. [34]. In the case of the empty (transparent) areas and the black stars we fit also the source count distribution $d N / d F$ from the 3FGL catalog. Note that black dots and black star overlap and cannot be visibly separated. For each set of contours, the inner one indicates the $68 \%$ C.L. region and outer one the $95 \%$ C.L. region.

PL. They follow quite closely the case with only APS data, apart from the PL of $\gamma_{1}$ that extends until 0.25 at $95 \%$ C.L. Figure 9 compares the results of this scan with the data sets used. Since including the $d N / d F$ does not change the best-fit solution, the solid blue lines in Fig. 9 are very similar to the solid red lines in Fig. 8, with no underestimate of the autocorrelation APS below $1 \mathrm{GeV}$ but a systematic underestimate of the $d N / d F$ above $3-4 \times 10^{-8} \mathrm{~cm}^{-2} \mathrm{~s}^{-1}$. This indicates that the likelihood is dominated by the APS measurement. The $\chi^{2}$ of the best-fit solution is 166.44 , corresponding to a $\chi^{2}$ per degree of freedom of 2.52 and a $p$-value of $10^{-10}$.

As in the previous section, we extend our model by including an additional source class, parametrized as in Eq. (12) by $C_{\mathrm{P}}^{0,0}$ and $\Gamma_{\text {new }}$, for which we consider the same prior ranges as before. The two-dimensional PL for the model parameter is shown in Fig. 7 by the green regions (fit to APS data only) and by the empty areas (fit to the APS and 3FGL $d N / d F$ data). The black circles and the black stars indicate the best-fit points, respectively. As in the previous section, the size of the contours increases with respect to Fig. 6 and they include the preferred regions in Fig. 6. They are also in qualitative agreement with Fig. 4, confirming that the data sets corresponding to the two masks point towards the same blazar population.
When the $d N / d F$ data are included (empty areas in Fig. 7) there is a shift of the preferred regions to lower values of $A$ (and, thus, larger values of $\gamma_{1}$ ). However, the two sets of contours are located along the same degeneracy. These regions correspond to solutions that are in a better agreement with the 3FGL $d N / d F$, as it can be seen by the blue dashed lines in Fig. 9: compared to the case with blazars only (blue solid lines in Fig. 9), the better agreement with the APS data provided by the new source class increases the weight of the $d N / d F$ data in the likelihood. Thus, the scan is strongly driven towards configurations that also provide a good description to the source count distribution.

When fitting the APS data alone (Fig. 8), the best-fit $\chi^{2}$ is 74.51 (best-fit $\chi^{2}$ per degree of freedom of 1.46 and $p$-value of 0.02). The likelihood-ratio test yields a $p$-value of $2 \times 10^{-10}$, corresponding to more than $10 \sigma$ evidence in favor of the new source class. The strong preference is confirmed also within a Bayesian framework, with a $\ln B$ of 20.93. When we include the 3FGL $d N / d F$ data in the fit (Fig. 9), the best-fit $\chi^{2}$ is 97.78 , corresponding to a best-fit $\chi^{2}$ per degree of freedom of 1.53 and to a $p$-value of $4 \times 10^{-3}$. The $p$-value of the likelihood-ratio test is $4 \times 10^{-16}$ and the Bayes factor $\ln B$ is 31.20. Both approaches strongly favor the presence of the new class. 

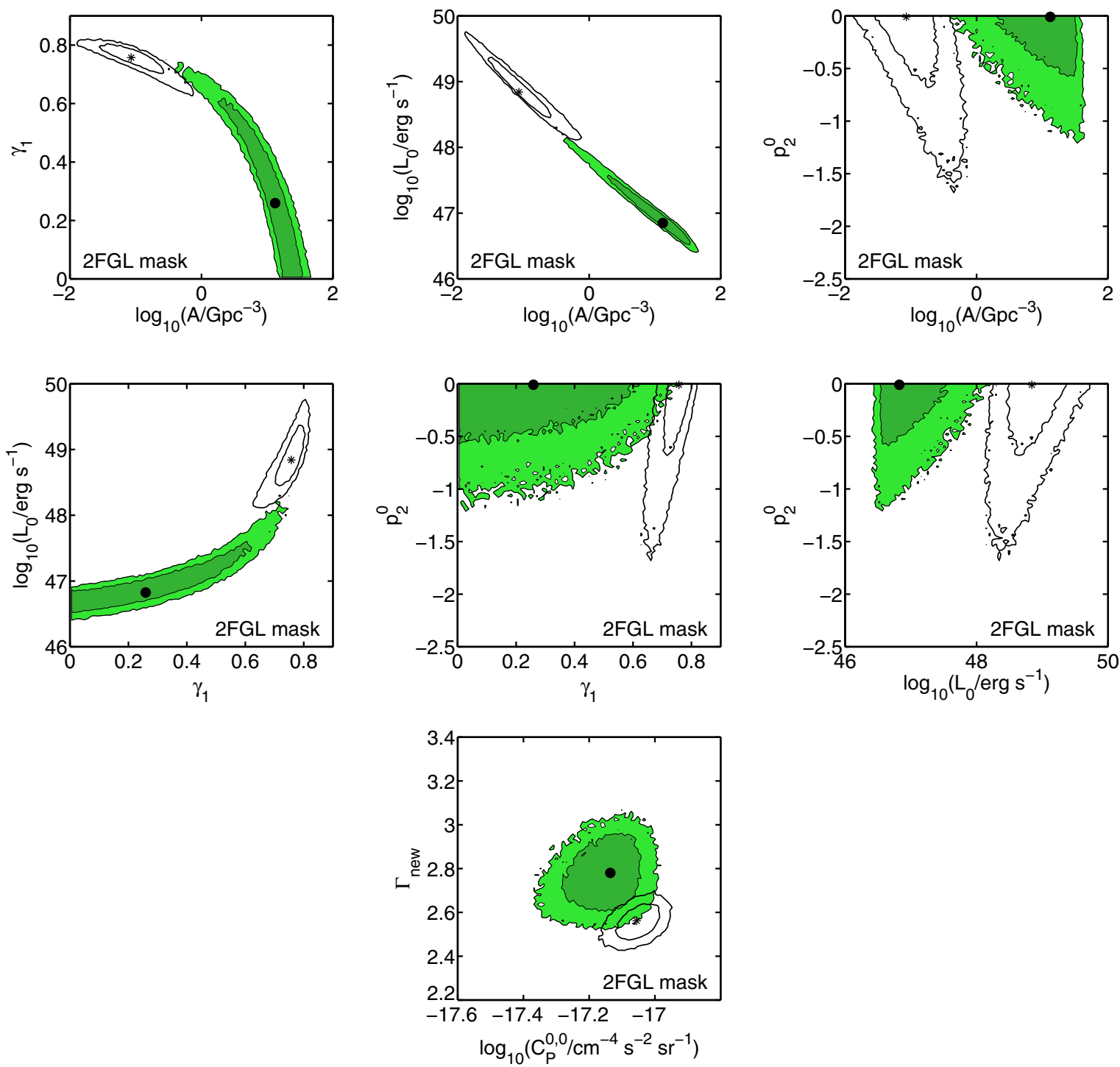

FIG. 7. Two-dimensional profile-likelihood contour plots for some combinations of parameters $A, \gamma_{1}, L_{0}, p_{2}^{0}, C_{\mathrm{P}}^{0,0}$ and $\Gamma_{\text {new }}$. The contours indicate the level of agreement between the model predictions and the data considered in the fit. The model includes the contribution of blazars and of the new source class, see Sec. IV D. The filled green contours and the black dots refer to a fit to the autoand cross-correlation APS from Ref. [34]. In the case of the empty (transparent) areas and the black stars we fit also the source count distribution $d N / d F$ from the 3FGL catalog. For each set of contours, the inner one indicates the $68 \%$ C.L. region and outer one.

Note that the best-fit values of $C_{\mathrm{P}}^{0,0}$ and $\Gamma_{\text {new }}$ (last panel of Fig. 7) are in agreement (at 95\% C.L.) with the values obtained when fitting the APS with the 3FGL mask (with and without the 3 FGL $d N / d F$ data, see Fig. 5). Indeed, the autocorrelation APS associated with the new source class (thin dash line in Figs. 2, 3, 8 and 9) is very similar independently of the mask used. This might suggest that there are almost no members of the new source class with fluxes between the sensitivities of the 3FGL and of 2FGL catalogs.

\section{DISCUSSION}

\section{A. Comparison with the EGB intensity energy spectrum}

The gray boxes in Fig. 10 denote the EGB intensity energy spectrum from Ref. [3] (model A). We compare it with the energy spectrum of the emission produced by all blazars (resolved and unresolved) according to the results of our scans. Top panels refer to the fits to the APS with the 3FGL mask (see Secs. IV A, IV B, and IV C), while the botton ones to the case of the 2FGL mask (see Sec. IV D). In the left panels we show results for fits to the APS data only, while in the right ones we also include the 3FGL $d N / d F$. Each panel contains two data sets, one for the case in which only blazars are considered in the model predictions (solid lines) and one for the fit including the new source class (dashed lines). Each data point is surrounded by its $68 \%$ C.L. (red or blue) and 95\% C.L. (pink or light blue) estimated error. Note that, even when the new source class is included in the fit, Fig. 10 only shows the emission of blazars. 

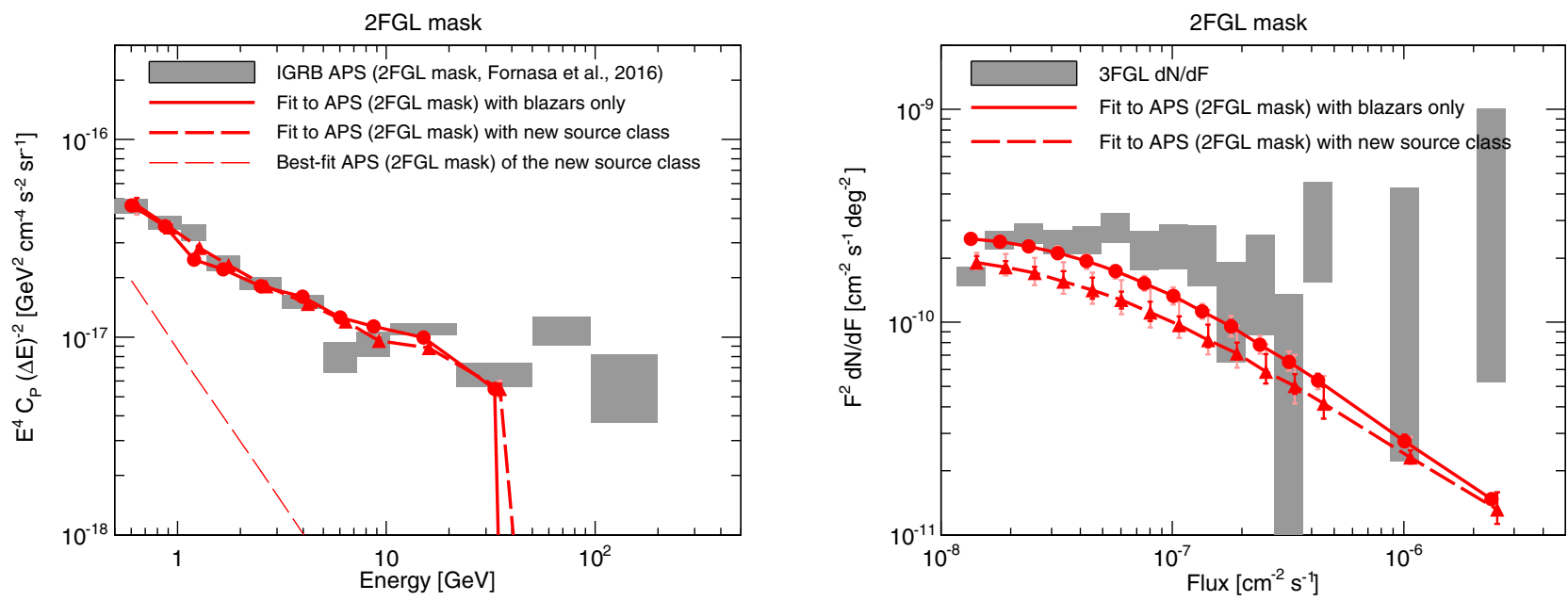

FIG. 8. The panels compare the results of our fits (red lines, cirlces and triangles) to different data sets (gray boxes). The left panel is for the autocorrelation APS with the 2FGL mask and the right panel is for the 3FGL $d N / d F$. For the fits considered here, only auto- and cross-correlation APS from Ref. [34] are included in the likelihood; see Sec. IV D. The thicker solid red lines and the red circles denote the best-fit solution for a model in which only blazars are included (no new source class). The thicker dashed red lines and the red triangles show the best-fit solution for a model including both blazars and the new source class. The thinner red dashed line in the left panel shows the best-fit autocorrelation APS for the new class source. Vertical error bars indicate the 68\% (in red) and 95\% C.L. (in pink) uncertainty. Circles and triangles are slightly shifted with respect to each other to increase readability.
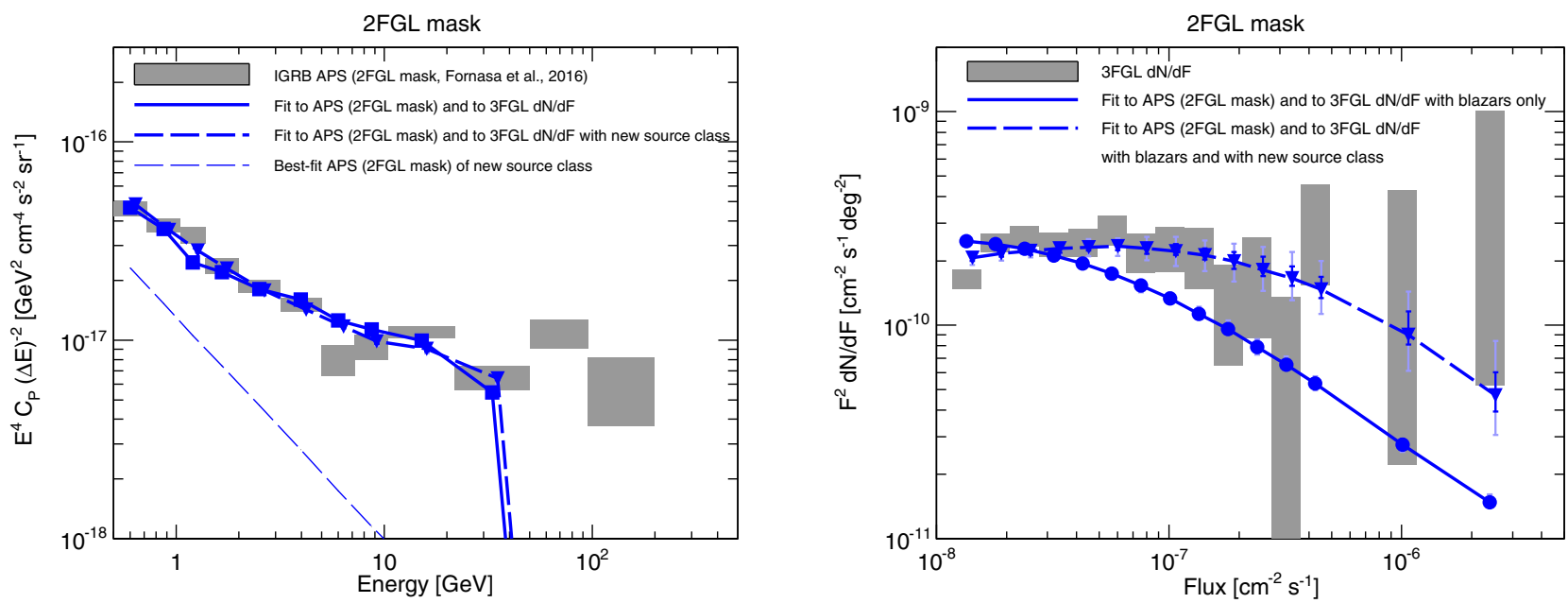

FIG. 9. The panels compare the results of our fits (blue lines, squares and triangles) to different data sets (gray boxes). The left panel is for the autocorrelation APS with the 2FGL mask and the right panel is for the 3FGL $d N / d F$. For the fits considered here the auto- and cross-correlation APS from Ref. [34] are included in the likelihood, as well as the 3FGL $d N / d F$. The thicker solid blue lines and the blue squares denote the best-fit solution for a model in which only blazars are included (no new source class). The thicker dashed blue lines and the blue triangles show the best-fit solution for a model including both blazars and the new source class. The thinner blue dashed line in the left panel shows the best-fit autocorrelation APS for the new class source. Vertical error bars indicate the $68 \%$ (in dark blue) and 95\% C.L. (in light cyan) uncertainty. Squares and triangles are slightly shifted with respect to each other to increase readability.

As pointed out in the previous sections, all the scans performed in terms of blazars only (no new sources) are in qualitative agreement (independent of which APS data are used or if the $d N / d F$ data are included). Indeed, the four solid lines in Fig. 10 are very similar. Blazars are responsible for a fraction of the EGB above $800 \mathrm{MeV}$ that goes from $45 \%$ to $49 \%$.
When we include the new source class (dashed lines), the emission is harder and dashed lines start to deviate from the solid ones around $80-100 \mathrm{GeV}$. This is probably because, since the new class account for the low-energy regime, blazars are more tuned to reproduce the behavior at high energies. In the best-fit scenarios, blazars account for between $43 \%$ and $57 \%$ of the EGB above $800 \mathrm{MeV}$. 

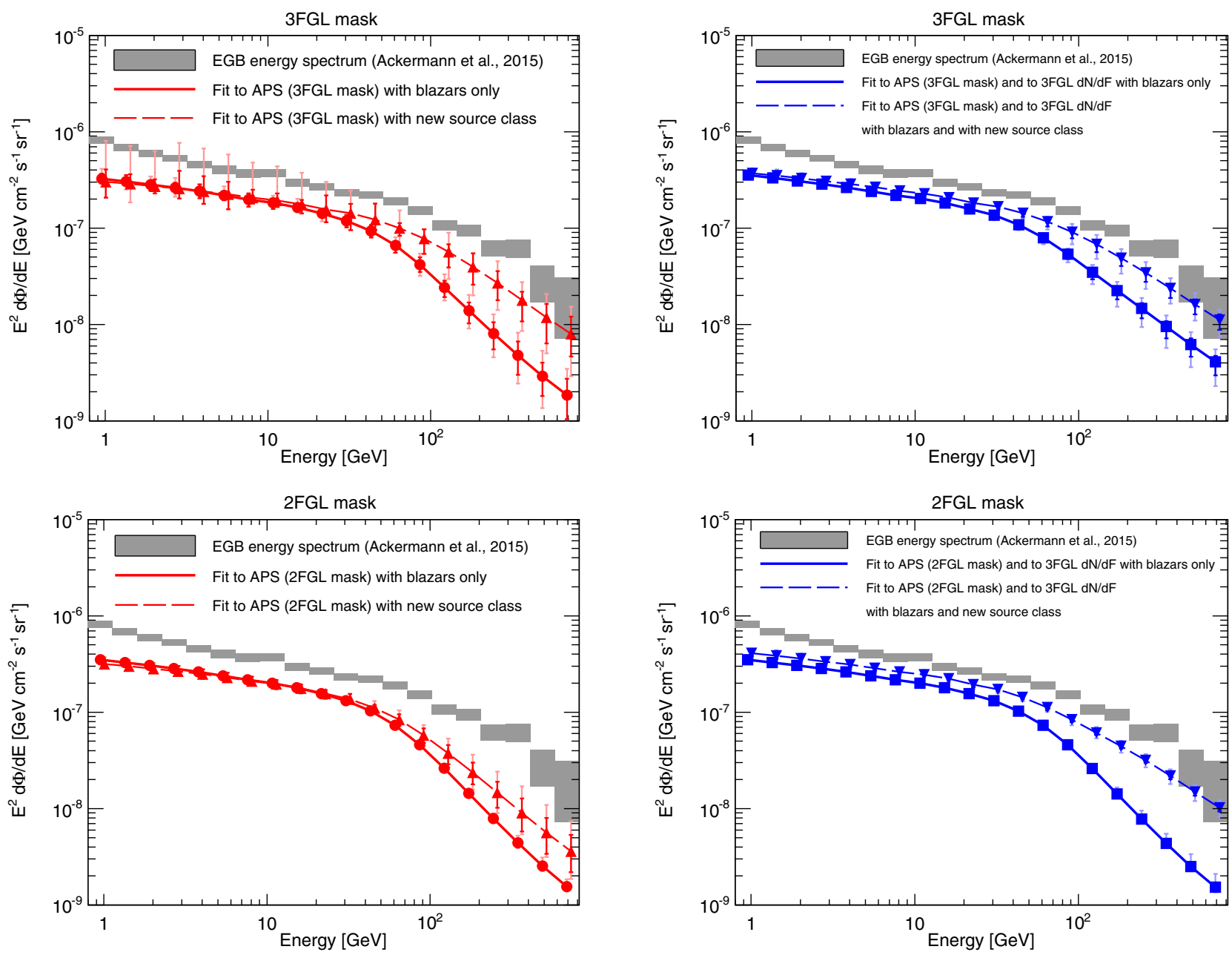

FIG. 10. The gray boxes indicate the EGB intensity energy spectrum measured in Ref. [3] (model A) and the colored line the predicted EGB intensity spectrum for the different scans performed. (Top-left panel): The scans are performed only fitting auto- and crosscorrelation APS from Ref. [34] (3FGL mask). The solid red line and red circles refer to the case with only blazars in the model (see Sec. IV A) and the dashed red line and the red triangles refer to the case in which the contribution of the new source class is included (see Sec. IV C). (Top-right panel): The same quantities are plotted, as in the top-left panel, but the fit is to the APS data (3FGL mask) and the $3 F G L d N / d F$ (see Secs. IV B and IV C). (Bottom panels): the same information as in the in the top panels is plotted, but the data fitted is the APS obtained with the 2FGL mask. In all panels, the red/blue bars around the markers indicate the $68 \%$ C.L. uncertainty, while the pink/light blue ones indicate the $95 \%$ C.L. one. The two sets of data in each panel are slightly shifted with respect to each other to increase readability.

\section{B. Characterization of the new source class}

We start by noting that a slope $\Gamma_{\text {new }}$ between approximately 2.5 and 3.2 (depending on the scan considered) is too soft to be compatible with the observed spectrum of star-forming galaxies [62,63] (at least at $\sim 1 \mathrm{GeV}$ ) and of misaligned active galactic nuclei [64,65] (see, however, Ref. [66]). Also, according to Ref. [12], these two classes of gamma-ray emitters are not expected to give rise to an APS as large as the one measured in Ref. [34] below $1 \mathrm{GeV}$.

Approximately one third of the sources in the 3FGL catalog are unassociated [67]. These emitters are good candidates to play the role of the new source class uncovered in our analysis. However, unassociated sources exhibit harder energy spectra than what we find in our scan.
The average slope of the 271 unassociated gamma-ray sources in the 3FGL catalog detected at $|b|>30^{\circ}$ is 2.26 .

The inferred energy spectrum of the new source class could be in agreement with the gamma-ray emission expected from the annihilation or decay of Dark Matter in Galactic and/or extragalactic halos and subhalos. For example, a Dark Matter candidate with a mass of the order of few $\mathrm{GeV}$ and annihilating into $b$ quarks would give rise to the desired energy spectrum. Many works in the literature have estimated the level of anisotropies expected for the gamma-ray emission induced by Dark Matter [5,6,34,68-75]. Ref. [34] employed a hybrid method, based on the results of $N$-body simulations and complemented by analytical estimates. In that case, the anisotropy signal due to Dark Matter is dominated by the contribution of Galactic 
subhalos. However, it would not be Poissonian, in contrast with the measured auto- and cross-APS used here.

In Ref. [34] the sub-GeV regime is where the measured auto- and cross-APS is potentialy affected by systematics related to the subtraction of the Galactic foreground, to leakage outside the mask or to specific details of data selection (see Sec. V-C of Ref. [34]). Even though it was tested that each of these effects cannot induce a deviation larger than $\sim 1 \sigma$ from the final data set, maybe the simultaneous presence of difference systamatics could have artificially enhanced the anisotropy expected from blazars to the level that is actually observed. This would reduce the need for the new class. The on-going measurement of gamma-ray anisotropies with Pass 8 Fermi LAT data will provide more information regarding to this scenario.

Given the difficulty to associate the new source class with any known population of gamma-ray emitters, we attempt a phenomenological description. We assume that the new sources are well described by a broken-power-law source count distribution:

$$
\frac{d N}{d F}=\left\{\begin{array}{ll}
N_{0} F^{-s} & \text { for } F<F_{0} \\
N_{0} F^{-2.5} & \text { for } F \geq F_{0}
\end{array} .\right.
$$

The flux in Eq. (13) is defined above $100 \mathrm{MeV}$. The index above the break $F_{0}$ is fixed to the Euclidean value, i.e. 2.5, typical of sources that are homogenously distributed in a local volume [76,77]. This is particularly appropriate for rare emitters, as for the large-flux end of the distribution. On the contrary, the slope $s$ below the break is left free between 0 and 2.5. We determine the normalization $N_{0}$ by requiring that the corresponding autoAPS in the first energy bins is equal to the measured APS (with the 3FGL mask) ${ }^{11}$ As in Sec. II, the APS is computed as the difference of $C_{\mathrm{P}, \mathrm{cov}=1}$ (defined, in this case, as the integral of $F^{2} d N / d F$ below the 3FGL sensitivity, taken from the last row of Table I, in order to select only unresolved sources) and $C_{\mathrm{P} \text {,cat }}$ (i.e. the APS of the sources in the 3FGL catalog, see Table I). Having determined $N_{0}$, we integrate $d N / d F$ for each value of $s$ and $F_{0}$ above the 3FGL sensitivity and above the 2FGL one to estimate the number of new sources expected in the 3FGL and in the 2FGL catalog, respectively. The two sensitivities are taken from the last rows of Table I and II. We also compute the expected APS between 0.50 and $0.72 \mathrm{GeV}$ and the integral of $F d N / d F$. Both quantities are computed below the 2FGL sensitivity in order to select the contribution of unresolved sources. They are, then, compared to the best-fit

\footnotetext{
${ }^{11} \mathrm{We}$ consider the best-fit value of $C_{\mathrm{P}}^{0,0}$ from the scan performed only with the APS data (3FGL mask); see Fig. 4. Also, since we want to reproduce the autocorrelation APS in the first energy bin from Ref. [34], the integration variable is now the flux between 0.50 and $0.72 \mathrm{GeV}$. We translate the flux above $100 \mathrm{MeV}$ used in Eq. (13) into the flux between 0.50 and $0.72 \mathrm{GeV}$ by assuming the best-fit value for $\Gamma_{\text {new }}$ from Fig. 4 .
}

APS of the new source class (for the 2FGL mask, see Sec. IV D) and to the IGRB intensity from Ref. [3].

For $0<s<2$, all the quantities mentioned above are well defined. We summarize the scenario in the left panel of Fig. 11: colors indicate the fraction of the observed IGRB (above $100 \mathrm{MeV}$ ) that can be explained by the new source class. Black lines indicate the regions where the fraction is $75 \%, 100 \%, 500 \%$ and $1000 \%$. The region below the black line labeled "100\%" is not viable, disfavoring values of $F_{0}$ smaller than approximately $2-3 \times 10^{-9} \mathrm{~cm}^{-2} \mathrm{~s}^{-1}$ and value of $s$ between 1.5 and 2.0. The blue (red) line indicates the combinations of $s$ and $F_{0}$ that predict 271 (168) sources above the 3FGL (2FGL) sensitivity, respectively. ${ }^{12}$ The regions above the blue and red lines would give rise to a number of new sources that are larger than the number of unassociated sources detected in the two catalogs for $|b|>30^{\circ}$. Those regions are, therefore, excluded. Finally, the green line indicates the combinations of $s$ and $F_{0}$ that correspond to a $C_{\mathrm{P}}^{0,0}$ of $9.5 \times 10^{-18} \mathrm{~cm}^{-4} \mathrm{~s}^{-2} \mathrm{sr}^{-1}$ when computed for the 2FGL mask. This value is the upper bound of the $95 \%$ C.L. interval for $C_{\mathrm{P}}^{0,0}$ in the fit to the APS data with the 2FGL mask (see Fig. 7). Thus, the region above the green line is also excluded as it would be incompatible at $95 \%$ C.L. with the results of that fit. This leaves a narrow region around $F_{0} \sim 4 \times 10^{-9} \mathrm{~cm}^{-2} \mathrm{~s}^{-1}$ and $s<1.5$, in which the new source class dominates the measured IGRB intensity.

For $s \geq 2$, the computation of the IGRB intensity diverges for $F \rightarrow 0$. Thus, we introduce a cut $F_{\text {lim }}$ below which we assume no new source is present. We consider two benchmark cases for $F_{\text {lim }}: 10 \%$ and $5 \%$ of the 3FGL sensitivity from Table I. The red lines in the right panel of Fig. 11 indicate the region where the new source class accounts for certain fractions of the observed IGRB, for the case of an $F_{\text {lim }}$ equal to $10 \%$ of the 3FGL sensitivity. The blue lines represent the same quantity but for an $F_{\text {lim }}$ equal to $5 \%$ of the 3FGL sensitivity. If we consider the higher $F_{\text {lim }}$ (i.e. red lines), the new source class never overshoots the measured IGRB above $100 \mathrm{Mev}$, accounting for, at the most, $\sim 82 \%$ of the emission. However, the gray region in the top left part of the panel is also not viable, as the new source class would predict too many sources in the 3FGL catalog. For the lower $F_{\lim }$ (i.e. blue lines), values of $F_{0}$ smaller than approximately $5 \times 10^{-9} \mathrm{~cm}^{-2} \mathrm{~s}^{-1}$ and of $s$ larger than 2.26 are excluded since the new gamma-ray emitters would overproduced the measured IGRB, above $100 \mathrm{MeV}$. The region above the green line is also not viable, as it corresponds to too many unassociated sources at high latitude in the 3FGL catalog. Then, the only allowed area is localized around $F_{0}=10^{-8} \mathrm{~cm}^{-2} \mathrm{~s}^{-1}$ and for $s<2.26$.

\footnotetext{
${ }^{12}$ The 3FGL and 2FGL sensitivities above $100 \mathrm{MeV}$ are assumed to be $10^{-18} \mathrm{~cm}^{-2} \mathrm{~s}^{-1}$ and $1.8 \times 10^{-18} \mathrm{~cm}^{-2} \mathrm{~s}^{-1}$, respectively. These value are taken from Fig. 8 of Ref. [67], for a spectral index of $\sim 3$, i.e. the best-fit point of $\Gamma_{\text {new }}$ from Fig. 5 .
} 

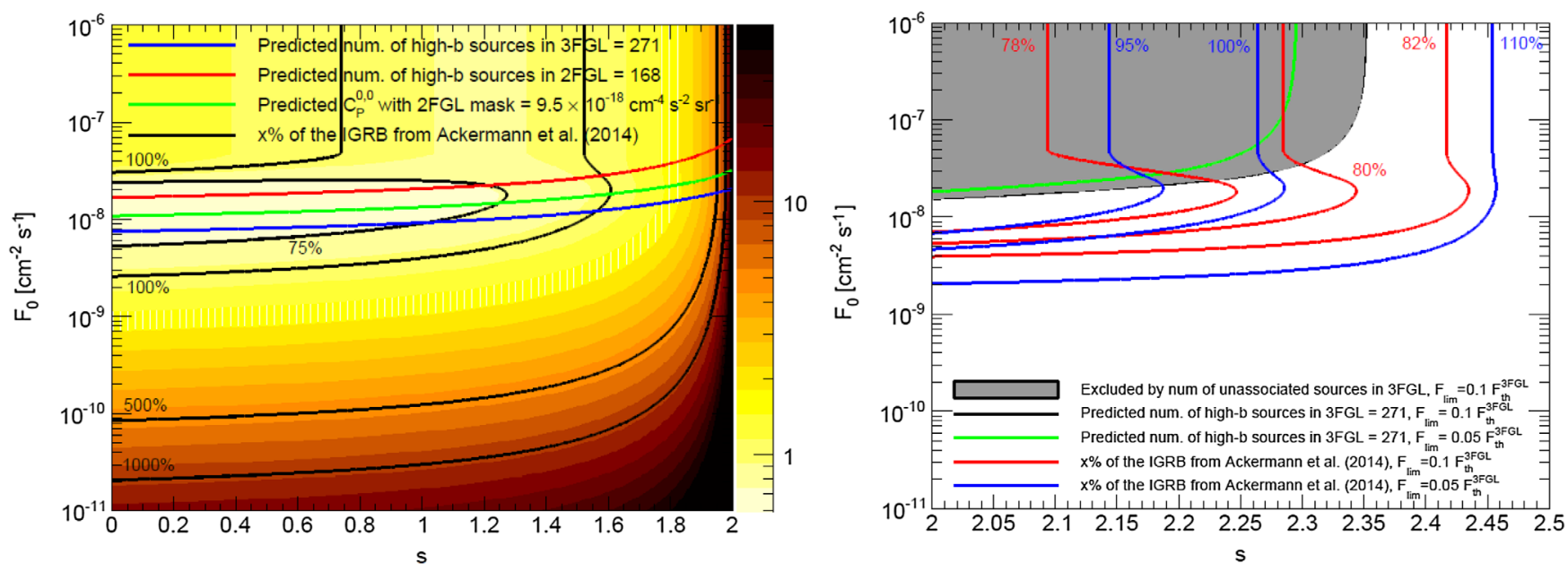

FIG. 11. The panels indicate the two-dimensional parameter space of the new source class, according to the model in Eq. (13). (Left panel:) The color bar shows what fraction of the measured IGRB intensity can be ascribed to the new source class (above $100 \mathrm{MeV}$ ). The black lines mark specific levels, indicated in the labels. The blue (red) line shows what combination of $s$ and $F_{0}$ correspond to a number of sources equal to 271 (168) above the 3FGL (2FGL) sensitivity and for $|b|>30^{\circ}$. The 3FGL and 2FGL sensitivities assumed here are $10^{-18} \mathrm{~cm}^{-2} \mathrm{~s}^{-1}$ and $1.8 \times 10^{-18} \mathrm{~cm}^{-2} \mathrm{~s}^{-1}$, respectively, above $100 \mathrm{MeV}$. The green line indicates where the new source class predicts a $C_{\mathrm{P}}^{0,0}$ of $9.5 \times 10^{-18} \mathrm{~cm}^{-4} \mathrm{~s}^{-2} \mathrm{sr}^{-1}$, below the 2FGL threshold, i.e. for unresolved sources. The regions above the colored lines is excluded, see text. (Right panel:) The red (blue) lines indicate what combinations of $s$ and $F_{0}$ correspond to specific fractions of the measured IGRB intensity above $100 \mathrm{MeV}$, for a $F_{\lim }=10 \%\left(F_{\lim }=5 \%\right)$ of the 3FGL sensitivity. The black line denotes where we would have 271 sources in the $3 \mathrm{FGL}$ catalog (with $|b|>30^{\circ}$ ) and the green one where we would have 168 sources in the $2 \mathrm{FGL}$ one (with $|b|>30^{\circ}$ ). The regions above these lines are, therefore, excluded.

\section{CONCLUSIONS}

In this work, we fit the recently published measurement of the IGRB anisotropy auto- and cross-APS with a physically motivated model of blazars. Reference [4] demonstrated that such a model provides a good description of the blazars observed by Fermi LAT. Here we use it to test whether blazars are able to reproduce the new APS measurement. A positive answer would confirm the result of Refs. $[9,10]$ (based on the original 2012 APS data [33]), according to which the IGRB APS is compatible with being due entirely to blazars. On the other hand, a negative answer would corroborate the phenomenological analysis performed in Ref. [34], establishing the need for more than one component to interpret IGRB anisotropies. We considered separately the APS measured with the "3FGL mask" and with the "2FGL mask" so that the APS is contributed only by gamma-ray emitters that are unresolved.

Our findings are summarized as follows:

(i) When fitting the new auto- and cross-APS (in the case of the 3FGL mask) in terms of blazars only, our best-fit solution is in agreement at $68 \%$ C.L. with the best fit obtained in Ref. [4], apart from our predicted $p_{2}^{0}$, which is larger. Including the $3 \mathrm{FGL} d N / d F$ in the fit does not have a significant impact.

(ii) Blazars alone (with or without including the 3FGL $d N / d F$ in the fit) underproduce the auto- and crossAPS observed with the 3FGL mask below $1 \mathrm{GeV}$. This suggests that a different class of gamma-ray emitters is needed to reproduce the measured APS. Note that previous works analysing the IGRB anisotropies in terms of blazars (as, e.g., Refs. $[9,10]$ ) could not be sensitive to this new source class as they were based on the 2012 APS measurement from Ref. [33], which did not extend below $1 \mathrm{GeV}$. Our result validates the findings of Ref. [34] and it suggests that sub-GeV anisotropies are due (at least in part) to gamma-ray emitters with a soft spectrum (with values of $\Gamma_{\text {new }}$ ranging from 2.7 to 3.2). The properties of the new sources are consistent, whether we include the 3FGL $d N / d F$ data in the fit or not. By a likelihood-ratio test, the new source is preferred over the blazar-only scenario by, at least, $5 \sigma$.

(iii) If we fit the APS obtained with the 2FGL mask with blazars only, our best-fit solution does not underproduce the sub-GeV APS, as before. A new source class still improves the fit to the data and it is preferred over the blazar-only scenario by more than $10 \sigma$. However, this new source class is different from the one hinted at in Ref. [34]. In fact, in Ref. [34], the APS below 2-3 GeV (with the 2FGL mask) is almost entirely due to the population with the lower energy break (in the scenario with the sources emitting as broken power laws, i.e. the description with the lowest $\chi^{2}$ per degree of freedom). ${ }^{13}$ While, in our

\footnotetext{
${ }^{13}$ Private communication.
} 
Figs. 8 and 9 the new class is always subdominant. This indicates that the class that is responsible for the low-energy data in Ref. [34] is probably a mixture of different gamma-ray emitters, including blazars. This also attests the benefit of using a physically motivated description of sources as we do here, instead of the phenomenological analysis performed in Ref. [34].

(iv) When we include the new source class, the 95\% C.L. contours point towards different regions of the parameter space, according to whether the 3FGL $d N / d F$ data are included or not in the fit. In particular, in order to achieve a good description of the 3FGL source count distribution, $\gamma_{1}$ needs to be of the order of 0.75 .

(v) The auto- and cross-correlation APS predicted by the new source class is very similar, independent of whether the scan is performed with or without the 3FGL $d N / d F$ data, with the 2FGL or 3FGL mask. It dominates the signal below $1 \mathrm{GeV}$ in the case of the 3FGL mask and it plays a subdominant role for the 2FGL mask. This implies that only a few members of the new source class are present with a flux between the 3FGL and the 2FGL sensitivity. The slope of the energy spectrum $\Gamma_{\text {new }}$ goes from 2.5 to 3.2 .

(vi) The properties of the new class inferred from the fit to the APS data disagree with the characteristics of known gamma-ray emitters, e.g., star-forming galaxies or misaligned active galactic nuclei. Also, unassociated sources in the 3FGL and 2FGL catalogs have, on average, harder energy spectra. Dark Matter halos and subhalos can reproduce the properties of the new source class (especially for a DarkMatter candidate with a mass of few $\mathrm{GeV}$ and annihilating into $b$ quarks). However, the expected APS would probably not be Poissonian, as assumed here. Finally, the combination of different systematic effects (i.e. contamination from Galactic foreground, leakage outside the mask and data selection) could enhance the auto- and cross-APS predicted by blazars below $\sim 1 \mathrm{GeV}$, improving the agreement with the data from Ref. [34] and reducing the need for the new source class.

(vii) We assume that the new gamma-ray emitters are characterized by a source count distribution that follows a broken power law. We leave the position of the break $F_{0}$ and the low-flux index $s$ as free parameters. In order to reproduce the APS measurement from Ref. [34], without, at the same time, overshooting the number of unassociated sources in the 2FGL and 3FGL catalog or the IGRB emission observed in Ref. [3], only values of $F_{0}$ around $4 \times 10^{-9} \mathrm{~cm}^{-2} \mathrm{~s}^{-1}$ (above $100 \mathrm{MeV}$ ) are allowed, for $s<1.5$. Alternatively, assuming that there is no source belonging to the new class below a $F_{\text {lim }}$ that is $10 \%$ of the 3FGL threshold, all considered values of $F_{0}$ and $s$ are allowed, apart from $F_{0}>$ $3 \times 10^{-8} \mathrm{~cm}^{-2} \mathrm{~s}^{-1}$ and $2<s<2.35$. On the other hand, if we lower $F_{\lim }$ to $5 \%$ of the 3FGL threshold, the only viable region is around $F_{0}$ of $10^{-8} \mathrm{~cm}^{-2} \mathrm{~s}^{-1}$ and $2<s<2.27$. In all cases, the new source class would be the dominant component to the IGRB intensity.

The amount of information that we have been able to extract on the IGRB from the new APS data attests the improvement that such a measurement represents, with respect to the original 2012 one. However, it is very challenging to achieve a coherent and consistent description of the IGRB by employing only one data set. We believe that the path to conclusively dissecting the composition of the IGRB lays in the combination of multiple complementary observables. Such a longer lever arm will also clarify the nature of the new class of sources suggested by the present work.

\section{ACKNOWLEDGMENTS}

S. A. and M. F. gratefully acknowledge support from the Netherlands Organization for Scientific Research (NWO) through a Vidi grant, and M.F. also thanks the project MultiDark CSD2009-00064. N.F., M.R. and H.S.Z. acknowledge support from the research grant Theoretical Astroparticle Physics No. 2012CPPYP7 under the program PRIN 2012 funded by the Ministero dell'Istruzione, dell'Università e della Ricerca (MIUR) and from the research grant TAsP (Theoretical Astroparticle Physics) funded by the Istituto Nazionale di Fisica Nucleare (INFN). M. R. acknowledges support from the "Excellent Young PI Grant: the Particle Dark-Matter Quest in the Extragalactic Sky" funded by the University of Torino and by Compagnia di San Paolo. H. S.Z. gratefully acknowledges INFN for a postdoctoral fellowship in theoretical physics on "Astroparticle, Dark Matter and Neutrino Physics," awarded under the INFN Fellowship Programme 2015. 
[1] F. Acero et al. (Fermi-LAT Collaboration), Astrophys. J. Suppl. Ser. 218, 23 (2015).

[2] M. Fornasa and M. A. Snchez-Conde, Phys. Rep. 598, 1 (2015).

[3] M. Ackermann et al. (Fermi-LAT Collaboration), Astrophys. J. 799, 86 (2015).

[4] M. Ajello et al., Astrophys. J. 800, L27 (2015).

[5] S. Ando, E. Komatsu, T. Narumoto, and T. Totani, Phys. Rev. D 75, 063519 (2007).

[6] S. Ando, E. Komatsu, T. Narumoto, and T. Totani, Mon. Not. R. Astron. Soc. 376, 1635 (2007).

[7] S. Ando and V. Pavlidou, Mon. Not. R. Astron. Soc. 400, 2122 (2009).

[8] J. M. Siegal-Gaskins, R. Reesman, V. Pavlidou, S. Profumo, and T. P. Walker, Mon. Not. R. Astron. Soc. 415, 1074 (2011).

[9] A. Cuoco, E. Komatsu, and J. M. Siegal-Gaskins, Phys. Rev. D 86, 063004 (2012).

[10] J. P. Harding and K. N. Abazajian, J. Cosmol. Astropart. Phys. 11 (2012) 026.

[11] F. Calore, M. Di Mauro, and F. Donato, Astrophys. J. 796, 14 (2014).

[12] M. Di Mauro, A. Cuoco, F. Donato, and J. M. SiegalGaskins, J. Cosmol. Astropart. Phys. 11 (2014) 021.

[13] D. Malyshev and D. W. Hogg, Astrophys. J. 738, 181 (2011).

[14] M. R. Feyereisen, S. Ando, and S. K. Lee, J. Cosmol. Astropart. Phys. 09 (2015) 027.

[15] H.-S. Zechlin, A. Cuoco, F. Donato, N. Fornengo, and A. Vittino, Astrophys. J. Suppl. Ser. 225, 18 (2016).

[16] M. Lisanti, S. Mishra-Sharma, L. Necib, and B. R. Safdi, Astrophys. J. 832, 117 (2016).

[17] H.-S. Zechlin, A. Cuoco, F. Donato, N. Fornengo, and M. Regis, Astrophys. J. 826, L31 (2016).

[18] J.-Q. Xia, A. Cuoco, E. Branchini, M. Fornasa, and M. Viel, Mon. Not. R. Astron. Soc. 416, 2247 (2011).

[19] J.-Q. Xia, A. Cuoco, E. Branchini, and M. Viel, Astrophys. J. Suppl. Ser. 217, 15 (2015).

[20] A. Cuoco, J.-Q. Xia, M. Regis, E. Branchini, N. Fornengo, and M. Viel, Astrophys. J. Suppl. Ser. 221, 29 (2015).

[21] M. Regis, J.-Q. Xia, A. Cuoco, E. Branchini, N. Fornengo, and M. Viel, Phys. Rev. Lett. 114, 241301 (2015).

[22] S. Ando, A. Benoit-Lvy, and E. Komatsu, Phys. Rev. D 90, 023514 (2014).

[23] S. Ando, J. Cosmol. Astropart. Phys. 10 (2014) 061.

[24] M. Shirasaki, S. Horiuchi, and N. Yoshida, Phys. Rev. D 92 , 123540 (2015).

[25] S. Ando and K. Ishiwata, J. Cosmol. Astropart. Phys. 06 (2016) 045.

[26] E. Branchini, S. Camera, A. Cuoco, N. Fornengo, M. Regis, M. Viel, and J.-Q. Xia, Astrophys. J. Suppl. Ser. 228, 8 (2017).

[27] S. Camera, M. Fornasa, N. Fornengo, and M. Regis, Astrophys. J. 771, L5 (2013).

[28] S. Camera, M. Fornasa, N. Fornengo, and M. Regis, J. Cosmol. Astropart. Phys. 06 (2015) 029.

[29] M. Shirasaki, S. Horiuchi, and N. Yoshida, Phys. Rev. D 90, 063502 (2014).

[30] M. Shirasaki, O. Macias, S. Horiuchi, S. Shirai, and N. Yoshida, Phys. Rev. D 94, 063522 (2016).
[31] T. Trster et al., Mon. Not. R. Astron. Soc. 467, 2706 (2017).

[32] N. Fornengo, L. Perotto, M. Regis, and S. Camera, Astrophys. J. 802, L1 (2015).

[33] M. Ackermann et al. (Fermi-LAT Collaboration), Phys. Rev. D 85, 083007 (2012).

[34] M. Fornasa et al., Phys. Rev. D 94, 123005 (2016).

[35] A. A. Abdo et al. (Fermi-LAT Collaboration), Astrophys. J. 720, 435 (2010).

[36] M. Ajello et al., Astrophys. J. 751, 108 (2012).

[37] M. Ajello et al., Astrophys. J. 780, 73 (2014).

[38] G. Fossati, L. Maraschi, A. Celotti, A. Comastri, and G. Ghisellini, Mon. Not. R. Astron. Soc. 299, 433 (1998).

[39] G. Ghisellini, A. Celotti, G. Fossati, L. Maraschi, and A. Comastri, Mon. Not. R. Astron. Soc. 301, 451 (1998).

[40] Y. Ueda, M. Akiyama, K. Ohta, and T. Miyaji, Astrophys. J. 598, 886 (2003).

[41] G. Hasinger, T. Miyaji, and M. Schmidt, Astron. Astrophys. 441, 417 (2005).

[42] T. Narumoto and T. Totani, Astrophys. J. 643, 81 (2006).

[43] Y. Inoue and T. Totani, Astrophys. J. 702, 523 (2009); 728, 73 (2011).

[44] K. N. Abazajian, S. Blanchet, and J. P. Harding, Phys. Rev. D 84, 103007 (2011).

[45] P. A. R. Ade et al. (Planck Collaboration), Astron. Astrophys. 571, A16 (2014).

[46] M. Ackermann et al. (Fermi-LAT Collaboration), Astrophys. J. 743, 171 (2011).

[47] G. Ghisellini, L. Maraschi, and F. Tavecchio, Mon. Not. R. Astron. Soc. 396, L105 (2009).

[48] E. T. Meyer, G. Fossati, M. Georganopoulos, and M. L. Lister, Astrophys. J. 752, L4 (2012).

[49] A. A. Abdo (Fermi-LAT Collaboration), Astrophys. J. 715, 429 (2010).

[50] A. Dominguez et al., Mon. Not. R. Astron. Soc. 410, 2556 (2011).

[51] A. Cooray and R. K. Sheth, Phys. Rep. 372, 1 (2002).

[52] P. L. Nolan et al. (Fermi-LAT Collaboration), Astrophys. J. Suppl. Ser. 199, 31 (2012).

[53] J. Buchner, A. Georgakakis, K. Nandra, L. Hsu, C. Rangel, M. Brightman, A. Merloni, M. Salvato, J. Donley, and D. Kocevski, Astron. Astrophys. 564, A125 (2014).

[54] F. Feroz and M. P. Hobson, Mon. Not. R. Astron. Soc. 384, 449 (2008).

[55] F. Feroz, M. P. Hobson, and M. Bridges, Mon. Not. R. Astron. Soc. 398, 1601 (2009).

[56] F. Feroz, M. P. Hobson, E. Cameron, and A. N. Pettitt, arXiv:1306.2144.

[57] R. Trotta, F. Feroz, M. P. Hobson, L. Roszkowski, and R. Ruiz de Austri, J. High Energy Phys. 12 (2008) 024.

[58] F. Feroz, K. Cranmer, M. Hobson, R. Ruiz de Austri, and R. Trotta, J. High Energy Phys. 06 (2011) 042.

[59] H. Chernoff and L. E. L., Ann. Math. Stat. 25, 579 (1954).

[60] A. Shapiro, Intl. Stat. Rev. 56, 49 (1988).

[61] R. Trotta, Contemp. Phys. 49, 71 (2008).

[62] M. Ackermann et al. (Fermi-LAT Collaboration), Astrophys. J. 755, 164 (2012).

[63] T. Linden, arXiv:1612.03175.

[64] M. Di Mauro, F. Calore, F. Donato, M. Ajello, and L. Latronico, Astrophys. J. 780, 161 (2014). 
[65] D. Hooper, T. Linden, and A. Lopez, J. Cosmol. Astropart. Phys. 08 (2016) 019.

[66] A. M. Brown, C. Boehm, J. Graham, T. Lacroix, P. M. Chadwick, and J. Silk, arXiv:1603.05469.

[67] M. Ackermann et al. (Fermi-LAT Collaboration), Astrophys. J. 810, 14 (2015).

[68] S. Ando and E. Komatsu, Phys. Rev. D 73, 023521 (2006).

[69] A. Cuoco, J. Brandbyge, S. Hannestad, T. Haugboelle, and G. Miele, Phys. Rev. D 77, 123518 (2008).

[70] J. M. Siegal-Gaskins, J. Cosmol. Astropart. Phys. 10 (2008) 040.

[71] J. M. Siegal-Gaskins and V. Pavlidou, Phys. Rev. Lett. 102, 241301 (2009).
[72] J. Zavala, V. Springel, and M. Boylan-Kolchin, Mon. Not. R. Astron. Soc. 405, 593 (2010).

[73] A. Cuoco, A. Sellerholm, J. Conrad, and S. Hannestad, Mon. Not. R. Astron. Soc. 414, 2040 (2011).

[74] M. Fornasa, J. Zavala, M. A. Sanchez-Conde, J. M. SiegalGaskins, T. Delahaye, F. Prada, M. Vogelsberger, F. Zandanel, and C. S. Frenk, Mon. Not. R. Astron. Soc. 429, 1529 (2013).

[75] S. Ando and E. Komatsu, Phys. Rev. D 87, 123539 (2013).

[76] J. A. Peacock, Cosmological Physics (Cambridge University Press, Cambridge, England, 1998).

[77] S. Ando, M. R. Feyereisen, and M. Fornasa, Phys. Rev. D 95, 103003 (2017). 